\title{
Rigidity and Vanishing Theorems for Almost Even-Clifford Hermitian Manifolds
}

\author{
Ana Lucia GARCIA-PULIDO and Rafael HERRERA \\ Centro de Investigación en Matemáticas, A. P. 402, Guanajuato, Gto., C.P. 36000, México \\ E-mail: lucia@cimat.mx,rherrera@cimat.mx \\ URL: https://sites.google.com/site/algarciapulido
}

Received October 10, 2016, in final form April 19, 2017; Published online April 23, 2017

https://doi.org/10.3842/SIGMA.2017.027

\begin{abstract}
We prove the rigidity and vanishing of several indices of "geometrically natural" twisted Dirac operators on almost even-Clifford Hermitian manifolds admitting circle actions by automorphisms.
\end{abstract}

Key words: almost even-Clifford Hermitian manifolds; index of elliptic operator; twisted Dirac operators; circle action by automorphisms

2010 Mathematics Subject Classification: 53C10; 53C15; 53C25; 58J20; 57S15

\section{Introduction}

There are two classical vanishing theorems for the $\widehat{A}$-genus (the index of the Dirac operator) on Spin manifolds: the Lichnerowicz vanishing [17] which assumes a metric of positive scalar curvature, and the Atiyah-Hirzebruch vanishing [3] which assumes smooth circle action. These vanishings can be seen and have been used frequently as obstructions to the existence of such metrics or actions. More vanishing theorems for the indices of $\mathrm{Spin}^{c}$ Dirac operators were explored by Hattori [11] on almost complex manifolds and $\mathrm{Spin}^{c}$ manifolds with compatible circle actions, which have parallels on complex manifolds with ample line bundles (a positivity condition for certain curvature) as in the case of the Kodaira vanishing theorem. Vanishing theorems have also been proven for indices of twisted Dirac operators on compact quaternion-Kähler manifolds with positive scalar curvature [16], and for almost quaternion-Hermitian manifolds with isometric circle actions that preserve the almost quaternion-Hermitian structure [12].

The vanishings of such indices on manifolds with isometric circle actions are instances of the rigidity of elliptic operators under such actions, an important property in the context of elliptic genera $[6,8,13,15,18,22,23,24]$. In this paper, we prove the rigidity and vanishing of the indices of several "geometrically natural" twisted Dirac operators on almost even-Clifford manifolds admitting circle actions by automorphisms, resembling those studied on almost quaternionicHermitian manifolds.

The note is organized as follows. In Section 2, we recall some material on Clifford algebras, Spin groups and representations, maximal tori of classical Lie groups, almost even-Clifford Hermitian manifolds and their structure groups. In Section 3, we examine the weights of the Spin representation in terms of the weights of the aforementioned structure groups and explore which representations to use in the twisted Dirac operators. In Section 4, we prove the vanishing Theorems 4.7, 4.8 and 4.9, using the Atiyah-Singer fixed point theorem.

\section{Preliminaries}

The material presented in this section can be consulted in $[1,7,9]$. 


\subsection{Clifford algebra, spin group and representation}

Let $\mathrm{Cl}_{n}$ denote the $2^{n}$-dimensional real Clifford algebra generated by the orthonormal vectors $e_{1}, e_{2}, \ldots, e_{n} \in \mathbb{R}^{n}$ subject to the relations

$$
e_{i} e_{j}+e_{j} e_{i}=-2 \delta_{i j},
$$

and $\mathbb{C l}_{n}=\mathrm{Cl}_{n} \otimes_{\mathbb{R}} \mathbb{C}$ its complexification. The even Clifford subalgebra $\mathrm{Cl}_{r}^{0}$ is defined as the invariant $(+1)$-subspace of the involution of $\mathrm{Cl}_{r}$ induced by the map $-\operatorname{Id}_{\mathbb{R}^{r}}$.

There exist algebra isomorphisms

$$
\mathbb{C l}_{n} \cong \begin{cases}\operatorname{End}\left(\mathbb{C}^{2^{k}}\right) & \text { if } n=2 k, \\ \operatorname{End}\left(\mathbb{C}^{2^{k}}\right) \oplus \operatorname{End}\left(\mathbb{C}^{2^{k}}\right) & \text { if } n=2 k+1,\end{cases}
$$

and the space of (complex) spinors is defined to be

$$
\Delta_{n}:=\mathbb{C}^{2^{k}}=\underbrace{\mathbb{C}^{2} \otimes \cdots \otimes \mathbb{C}^{2}}_{k \text { times }} .
$$

The map

$$
\kappa: \mathbb{C l}_{n} \longrightarrow \operatorname{End}\left(\mathbb{C}^{2^{k}}\right)
$$

is defined to be either the aforementioned isomorphism for $n$ even, or the isomorphism followed by the projection onto the first summand for $n$ odd. In order to make $\kappa$ explicit, consider the following matrices

$$
\mathrm{Id}=\left(\begin{array}{cc}
1 & 0 \\
0 & 1
\end{array}\right), \quad g_{1}=\left(\begin{array}{cc}
i & 0 \\
0 & -i
\end{array}\right), \quad g_{2}=\left(\begin{array}{cc}
0 & i \\
i & 0
\end{array}\right), \quad T=\left(\begin{array}{cc}
0 & -i \\
i & 0
\end{array}\right) .
$$

In terms of the generators $e_{1}, \ldots, e_{n}$ of the Clifford algebra, $\kappa$ can be described explicitly as follows

$$
\begin{aligned}
& e_{1} \mapsto \mathrm{Id} \otimes \mathrm{Id} \otimes \cdots \otimes \mathrm{Id} \otimes \mathrm{Id} \otimes g_{1}, \\
& e_{2} \mapsto \mathrm{Id} \otimes \mathrm{Id} \otimes \cdots \otimes \mathrm{Id} \otimes \mathrm{Id} \otimes g_{2}, \\
& e_{3} \mapsto \mathrm{Id} \otimes \mathrm{Id} \otimes \cdots \otimes \mathrm{Id} \otimes g_{1} \otimes T, \\
& e_{4} \mapsto \mathrm{Id} \otimes \mathrm{Id} \otimes \cdots \otimes \mathrm{Id} \otimes g_{2} \otimes T, \\
& \cdots \cdots \cdots \cdots \cdots \cdots \cdots \cdots \cdots \cdots \cdot, \\
& e_{2 k-1} \mapsto g_{1} \otimes T \otimes \cdots \otimes T \otimes T \otimes T, \\
& e_{2 k} \mapsto g_{2} \otimes T \otimes \cdots \otimes T \otimes T \otimes T,
\end{aligned}
$$

and, if $n=2 k+1$,

$$
e_{2 k+1} \mapsto i T \otimes T \otimes \cdots \otimes T \otimes T \otimes T .
$$

The vectors

$$
u_{+1}=\frac{1}{\sqrt{2}}(1,-i) \quad \text { and } \quad u_{-1}=\frac{1}{\sqrt{2}}(1, i),
$$

form a unitary basis of $\mathbb{C}^{2}$ with respect to the standard Hermitian product. Thus,

$$
\mathcal{B}=\left\{u_{\varepsilon_{1}, \ldots, \varepsilon_{k}}=u_{\varepsilon_{1}} \otimes \cdots \otimes u_{\varepsilon_{k}} \mid \varepsilon_{j}= \pm 1, j=1, \ldots, k\right\},
$$

is a unitary basis of $\Delta_{n}=\mathbb{C}^{2^{k}}$ with respect to the naturally induced Hermitian product. 
The Spin group $\operatorname{Spin}(n) \subset \mathrm{Cl}_{n}$ is the subset

$$
\operatorname{Spin}(n)=\left\{x_{1} x_{2} \cdots x_{2 l-1} x_{2 l}\left|x_{j} \in \mathbb{R}^{n},\right| x_{j} \mid=1, l \in \mathbb{N}\right\} \text {, }
$$

endowed with the product of the Clifford algebra. It is a Lie group and its Lie algebra is

$$
\mathfrak{s p i n}(n)=\operatorname{span}\left\{e_{i} e_{j} \mid 1 \leq i<j \leq n\right\}
$$

The restriction of $\kappa$ to $\operatorname{Spin}(n)$ defines the Lie group representation

$$
\kappa_{n}:=\left.\kappa\right|_{\operatorname{Spin}(n)}: \operatorname{Spin}(n) \longrightarrow \operatorname{GL}\left(\Delta_{n}\right),
$$

which is, in fact, special unitary. We have the corresponding Lie algebra representation

$$
\kappa_{n_{*}}: \mathfrak{s p i n}(n) \longrightarrow \mathfrak{g l}\left(\Delta_{n}\right)
$$

Recall that the $\operatorname{Spin}$ group $\operatorname{Spin}(n)$ is the universal double cover of $\operatorname{SO}(n), n \geq 3$. For $n=2$ we consider $\operatorname{Spin}(2)$ to be the connected double cover of $\mathrm{SO}(2)$. The covering map will be denoted by

$$
\lambda_{n}: \operatorname{Spin}(n) \rightarrow \mathrm{SO}(n) \subset \mathrm{GL}\left(\mathbb{R}^{n}\right) .
$$

Its differential is given by $\lambda_{n_{*}}\left(e_{i} e_{j}\right)=2 E_{i j}$, where $E_{i j}=e_{i}^{*} \otimes e_{j}-e_{j}^{*} \otimes e_{i}$ is the standard basis of the skew-symmetric matrices, and $e^{*}$ denotes the metric dual of the vector $e$. Furthermore, we will abuse the notation and also denote by $\lambda_{n}$ the induced representation on the exterior algebra $\bigwedge^{*} \mathbb{R}^{n}$.

By means of $\kappa$, we have the Clifford multiplication

$$
\begin{aligned}
\mu_{n}: \mathbb{R}^{n} \otimes \Delta_{n} & \longrightarrow \Delta_{n}, \\
x \otimes \phi & \longmapsto \mu_{n}(x \otimes \phi)=x \cdot \phi:=\kappa(x)(\phi) .
\end{aligned}
$$

The Clifford multiplication $\mu_{n}$ is skew-symmetric with respect to the Hermitian product

$$
\left\langle x \cdot \phi_{1}, \phi_{2}\right\rangle=\left\langle\mu_{n}\left(x \otimes \phi_{1}\right), \phi_{2}\right\rangle=-\left\langle\phi_{1}, \mu_{n}\left(x \otimes \phi_{2}\right)\right\rangle=-\left\langle\phi_{1}, x \cdot \phi_{2}\right\rangle,
$$

is $\operatorname{Spin}(n)$-equivariant and can be extended to a $\operatorname{Spin}(n)$-equivariant map

$$
\begin{aligned}
\mu_{n}: \bigwedge^{*}\left(\mathbb{R}^{n}\right) \otimes \Delta_{n} & \longrightarrow \Delta_{n}, \\
\omega \otimes \psi & \longmapsto \omega \cdot \psi .
\end{aligned}
$$

When $n$ is even, we define the following involution

$$
\begin{aligned}
\Delta_{n} & \longrightarrow \Delta_{n}, \\
\psi & \longmapsto(-i)^{\frac{n}{2}} \operatorname{Vol}_{n} \cdot \psi,
\end{aligned}
$$

where $\operatorname{vol}_{n}=e_{1} \cdots e_{n}$. The \pm 1 eigenspace of this involution is denoted $\Delta_{n}^{ \pm}$. These spaces have equal dimension and are irreducible representations of $\operatorname{Spin}(n)$. Note that our definition differs from the one given in [9] by a $(-1)^{\frac{n}{2}}$. The reason for this difference is that we want the spinor $u_{1, \ldots, 1}$ to be always positive. In this case, we will denote the two representations by

$$
\kappa_{n}^{ \pm}: \operatorname{Spin}(n) \longrightarrow \mathrm{GL}\left(\Delta_{n}^{ \pm}\right)
$$


For future use, let us recall the effect of $\operatorname{vol}_{n}$ on $\Delta_{n}=\Delta_{n}^{+} \oplus \Delta_{n}^{-}$when $n$ is even:

\begin{tabular}{|c|c|c|}
\hline$n(\bmod 8)$ & $\Delta_{n}^{+}$ & $\Delta_{n}^{-}$ \\
\hline 0 & 1 & -1 \\
\hline 2 & $i$ & $-i$ \\
\hline 4 & -1 & 1 \\
\hline 6 & $-i$ & $i$ \\
\hline
\end{tabular}

Furthermore, for $n \equiv 0(\bmod 4), n \neq 4$,

$$
\operatorname{ker}\left(\kappa_{n}^{+}\right)= \begin{cases}\left\{1, \operatorname{vol}_{r}\right\} & \text { if } r \equiv 0(\bmod 8), \\ \left\{1,-\operatorname{vol}_{r}\right\} & \text { if } r \equiv 4(\bmod 8),\end{cases}
$$

and

$$
\operatorname{ker}\left(\kappa_{n}^{-}\right)= \begin{cases}\left\{1,-\operatorname{vol}_{r}\right\} & \text { if } r \equiv 0(\bmod 8), \\ \left\{1, \operatorname{vol}_{r}\right\} & \text { if } r \equiv 4(\bmod 8) .\end{cases}
$$

For $r$ even, let

$$
\mathbb{P S O}(r):=\frac{\mathrm{SO}(r)}{\left\{ \pm \operatorname{Id}_{r}\right\}} \cong \frac{\operatorname{Spin}(r)}{\left\{ \pm 1, \pm \operatorname{vol}_{r}\right\}}
$$

and for $r \equiv 0(\bmod 4)$ let

$$
\operatorname{Spin}_{ \pm}(r) \cong \frac{\operatorname{Spin}(r)}{\left\{1, \pm \operatorname{vol}_{r}\right\}}
$$

Note that we will always denote by 1 and $\operatorname{Id}_{r}$ the identity elements of $\operatorname{Spin}(r)$ and $\operatorname{SO}(r)$ respectively.

\section{$2.2 \quad$ Maximal tori}

\subsection{1 $\mathrm{SO}(n)$}

Recall that a maximal torus of $\mathrm{SO}(n)$ is given by

$$
\left(\begin{array}{ccccc}
\cos \left(\eta_{1}\right) & -\sin \left(\eta_{1}\right) & & & \\
\sin \left(\eta_{1}\right) & \cos \left(\eta_{1}\right) & & & \\
& & \ddots & & \\
& & & \cos \left(\eta_{n / 2}\right) & -\sin \left(\eta_{n / 2}\right) \\
& & & \sin \left(\eta_{n / 2}\right) & \cos \left(\eta_{n / 2}\right)
\end{array}\right)
$$

if $n$ is even, and

$$
\left(\begin{array}{cccccc}
\cos \left(\eta_{1}\right) & -\sin \left(\eta_{1}\right) & & & \\
\sin \left(\eta_{1}\right) & \cos \left(\eta_{1}\right) & & & \\
& & \ddots & & \\
& & & \cos \left(\eta_{[n / 2]}\right) & -\sin \left(\eta_{[n / 2]}\right) & \\
& & & \sin \left(\eta_{[n / 2]}\right) & \cos \left(\eta_{[n / 2]}\right) & 1
\end{array}\right)
$$

if $n$ is odd. 


\subsection{2 $\operatorname{Spin}(n)$}

Each one of the $2 \times 2$ rotation blocks is a transformation that can be achieved by using Clifford product. For instance the rotation

$$
\left(\begin{array}{ccccc}
\cos \left(\varphi_{1}\right) & -\sin \left(\varphi_{1}\right) & & & \\
\sin \left(\varphi_{1}\right) & \cos \left(\varphi_{1}\right) & & & \\
& & 1 & & \\
& & & \ddots & \\
& & & & 1
\end{array}\right)
$$

can be achieved by using the element

$$
e_{1}\left(-\cos \left(\varphi_{1} / 2\right) e_{1}+\sin \left(\varphi_{1} / 2\right) e_{2}\right)=\cos \left(\varphi_{1} / 2\right)+\sin \left(\varphi_{1} / 2\right) e_{1} e_{2} \in \operatorname{Spin}(n)
$$

as follows

$$
\begin{aligned}
& \left(\cos \left(\varphi_{1} / 2\right)+\sin \left(\varphi_{1} / 2\right) e_{1} e_{2}\right) y\left(\cos \left(\varphi_{1} / 2\right)+\sin \left(\varphi_{1} / 2\right) e_{2} e_{1}\right) \\
& \quad=\left(y_{1} \cos \left(\varphi_{1}\right)-y_{2} \sin \left(\varphi_{1}\right)\right) e_{1}+\left(y_{1} \sin \left(\varphi_{1}\right)+y_{2} \cos \left(\varphi_{1}\right)\right) e_{2}+y_{3} e_{3}+\cdots+y_{n} e_{n}
\end{aligned}
$$

for $y=y_{1} e_{1}+\cdots+y_{n} e_{n} \in \mathbb{R}^{n}$. Thus, we see that the corresponding elements in $\operatorname{Spin}(n)$ are

$$
\pm\left(\cos \left(\varphi_{1} / 2\right)+\sin \left(\varphi_{1} / 2\right) e_{1} e_{2}\right) .
$$

Furthermore, we see that a maximal torus of $\operatorname{Spin}(n)$ is given by elements of the form

$$
t\left(\varphi_{1}, \ldots, \varphi_{k}\right)=\prod_{j=1}^{k}\left(\cos \left(\varphi_{j} / 2\right)+\sin \left(\varphi_{j} / 2\right) e_{2 j-1} e_{2 j}\right) .
$$

By using the explicit description (2.2) of the isomorphisms (2.1), we can check that

$$
t\left(\varphi_{1}, \ldots, \varphi_{k}\right) \cdot u_{\varepsilon_{1}, \ldots, \varepsilon_{k}}=e^{\frac{i}{2} \sum_{j=1}^{k} \varepsilon_{k+1-j} \varphi_{j}} \cdot u_{\varepsilon_{1}, \ldots, \varepsilon_{k}}
$$

i.e., the basis vectors $u_{\varepsilon_{1}, \ldots, \varepsilon_{k}}$ are weight vectors of the spin representation with weight

$$
\frac{1}{2} \sum_{j=1}^{k} \varepsilon_{k+1-j} \varphi_{j}
$$

which in coordinate vectors with respect to the basis $\left\{\varphi_{j}\right\}$ give the well known expressions

$$
\left( \pm \frac{1}{2}, \pm \frac{1}{2}, \ldots, \pm \frac{1}{2}\right)
$$

Indeed, in terms of the (appropriately ordered) basis $\mathcal{B}$, the matrix associated to an element $\left.t\left(\varphi_{1}, \ldots, \varphi_{\left[\frac{n}{2}\right]}\right]\right)$ is

$$
\left(\begin{array}{ccc}
e^{\frac{i}{2}\left(\varphi_{1}+\varphi_{2}+\cdots+\varphi_{\left[\frac{n}{2}\right]}\right)} & & \\
e^{\frac{i}{2}\left(-\varphi_{1}+\varphi_{2}+\cdots+\varphi_{\left[\frac{n}{2}\right]}\right)} & \\
e^{\frac{i}{2}\left(\varphi_{1}-\varphi_{2}+\cdots+\varphi_{\left[\frac{n}{2}\right]}\right)} & \\
\ddots & \\
& e^{\frac{i}{2}\left(-\varphi_{1}-\varphi_{2}+\cdots+\varphi_{\left[\frac{n}{2}\right]}\right)} \\
& \ddots & \\
& & e^{\frac{i}{2}\left(-\varphi_{1}-\varphi_{2}-\cdots-\varphi_{\left[\frac{n}{2}\right]}\right)}
\end{array}\right) .
$$


Note that, when $n$ is even, $\Delta_{n}^{+}$is generated by the basis vectors $u_{\varepsilon_{1}, \ldots, \varepsilon_{\frac{n}{2}}}$ with an even number of $\varepsilon_{j}$ equal to -1 , and $\Delta_{n}^{-}$is generated by the basis vectors $u_{\varepsilon_{1}, \ldots, \varepsilon_{n}}$ with an odd number of $\varepsilon_{j}$ equal to -1 . Therefore, after reordering the basis, the matrix above can be rearranged to have two diagonal blocks of equal size: one block in which the exponents contain an even number of negative signs

$$
\left(\begin{array}{c}
e^{\frac{i}{2}\left(\varphi_{1}+\varphi_{2}+\cdots+\varphi_{\frac{n}{2}}\right)} \\
e^{\frac{i}{2}\left(-\varphi_{1}-\varphi_{2}+\cdots+\varphi_{\frac{n}{2}}\right)} \\
e^{\frac{i}{2}\left(-\varphi_{1}+\varphi_{2}-\cdots+\varphi_{\frac{n}{2}}\right)} \\
\ddots
\end{array}\right)
$$

and another block in which the exponents contain an odd number of negative signs

$$
\left(\begin{array}{c}
e^{\frac{i}{2}\left(-\varphi_{1}+\varphi_{2}+\cdots+\varphi_{\frac{n}{2}}\right)} \\
e^{\frac{i}{2}\left(\varphi_{1}-\varphi_{2}+\cdots+\varphi_{\frac{n}{2}}\right)} \\
e^{\frac{i}{2}\left(\varphi_{1}+\varphi_{2}-\varphi_{3}+\cdots+\varphi_{\frac{n}{2}}\right)} \\
\ddots
\end{array}\right)
$$

\subsection{3 $\mathrm{U}(m)$}

The standard maximal torus of $\mathrm{U}(m)$ is

$$
\left(\begin{array}{cccc}
e^{i \theta_{1}} & & & \\
& e^{i \theta_{2}} & & \\
& & \ddots & \\
& & & e^{i \theta_{m}}
\end{array}\right)
$$

\subsection{4 $\operatorname{Sp}(m)$}

The standard maximal torus of $\operatorname{Sp}(m)$ is

$$
\left(\begin{array}{ccccc}
e^{i \theta_{1}} & & & & \\
& e^{-i \theta_{1}} & & & \\
& & \ddots & & \\
& & & e^{i \theta_{m}} & \\
& & & & e^{-i \theta_{m}}
\end{array}\right) \text {. }
$$

\subsection{Almost even-Clifford Hermitian structures}

Definition 2.1. Let $N \in \mathbb{N}$ and $\left(e_{1}, \ldots, e_{r}\right)$ an orthonormal frame of $\mathbb{R}^{r}$.

- A linear even-Clifford structure of rank $r$ on $\mathbb{R}^{N}$ is an algebra representation

$$
\Phi: \mathrm{Cl}_{r}^{0} \longrightarrow \operatorname{End}\left(\mathbb{R}^{N}\right) .
$$

- A linear even-Clifford Hermitian structure of rank $r$ on $\mathbb{R}^{N}$ (endowed with a positive definite inner product) is a linear even-Clifford structure of rank $r$ such that each bivector $e_{i} e_{j}, 1 \leq i<j \leq r$, is mapped to a skew-symmetric endomorphism $\Phi\left(e_{i} e_{j}\right)=J_{i j}$. 


\section{Remark 2.2.}

- Note that $J_{i j}^{2}=-\operatorname{Id}_{\mathbb{R}^{N}}$.

- Given a linear even-Clifford structure of rank $r$ on $\mathbb{R}^{N}$, we can average the standard inner product $\langle$,$\rangle on \mathbb{R}^{N}$ as follows

$$
(X, Y)=\sum_{k=1}^{[r / 2]}\left[\sum_{1 \leq i_{1}<\cdots<i_{2 k}<r}\left\langle\Phi\left(e_{i_{1} \ldots i_{2 k}}\right)(X), \Phi\left(e_{i_{1} \ldots i_{2 k}}\right)(Y)\right\rangle\right],
$$

where $\left(e_{1}, \ldots, e_{r}\right)$ is an orthonormal frame of $\mathbb{R}^{r}$, so that the linear even-Clifford structure is Hermitian with respect to the averaged inner product.

- Given a linear even-Clifford Hermitian structure of rank $r$, the subalgebra $\mathfrak{s p i n}(r)$ is mapped injectively into the skew-symmetric endomorphisms $\operatorname{End}^{-}\left(\mathbb{R}^{N}\right)$.

Definition 2.3. Let $r \geq 2$.

- A rank $r$ almost even-Clifford structure on a smooth manifold $M$ is a smoothly varying choice of a rank $r$ linear even-Clifford structure on each tangent space of $M$.

- A smooth manifold carrying an almost even-Clifford structure will be called an almost even-Clifford manifold.

- A rank $r$ almost even-Clifford Hermitian structure on a Riemannian manifold $M$ is a smoothly varying choice of a linear even-Clifford Hermitian structure on each tangent space of $M$.

- A Riemannian manifold carrying a rank $r$ almost even-Clifford Hermitian structure will be called a rank $r$ almost even-Clifford Hermitian manifold, or an almost- $\mathrm{Cl}_{r}^{0}$-Hermitian manifold for short.

Remark 2.4. Our definition of almost even-Clifford Hermitian structure does not require the existence of a Riemannian vector bundle of rank $r$. Therefore, it includes both the notions of even Clifford structure and projective even Clifford structure introduced in [19, Definition 2.2 and Remark 2.5].

\subsubsection{Structure groups of almost even-Clifford manifolds}

Thanks to [2], we know that the complexification of the tangent space of an almost- $\mathrm{Cl}_{r}^{0}$-Hermitian manifold decomposes as follows

\begin{tabular}{|c|c|}
\hline$r(\bmod 8)$ & $\mathbb{R}^{N} \otimes \mathbb{C}$ \\
\hline 0 & $\mathbb{C}^{m_{1}} \otimes \Delta_{r}^{+} \oplus \mathbb{C}^{m_{2}} \otimes \Delta_{r}^{-}$ \\
\hline 1,7 & $\mathbb{C}^{m} \otimes \Delta_{r}$ \\
\hline 2 & $\mathbb{C}^{m} \otimes \Delta_{r}^{+} \oplus \overline{\mathbb{C}^{m}} \otimes \Delta_{r}^{-}$ \\
\hline 6 & $\overline{\mathbb{C}^{m}} \otimes \Delta_{r}^{+} \oplus \mathbb{C}^{m} \otimes \Delta_{r}^{-}$ \\
\hline 3,5 & $\mathbb{C}^{2 m} \otimes \Delta_{r}$ \\
\hline 4 & $\mathbb{C}^{2 m_{2}} \otimes \Delta_{r}^{+} \oplus \mathbb{C}^{2 m_{1}} \otimes \Delta_{r}^{-}$ \\
\hline
\end{tabular}

where the different $\mathbb{C}^{p}$ denote the corresponding standard complex representations of the classical Lie groups $\mathrm{SO}(p), \mathrm{U}(p)$ or $\mathrm{Sp}(p)$. Note that the dimension of an almost even-Clifford Hermitian manifold depends of two or three parameters: the rank $r$ of the even-Clifford structure and the multiplicity $m$ or multiplicities $m_{1}, m_{2}$.

The structure groups of the aforementioned manifolds, for $r \geq 3$, are given as follows (see [1]): 
- For $r \not \equiv 0(\bmod 4)$

\begin{tabular}{|c|c|c|}
\hline$r(\bmod 8)$ & 0 & 1 \\
\hline 1,7 & $\frac{\mathrm{SO}(m) \times \operatorname{Spin}(r)}{\left\{ \pm\left(\operatorname{Id}_{m}, 1\right)\right\}}$ & $\mathrm{SO}(m) \times \operatorname{Spin}(r)$ \\
\hline 2,6 & $\frac{\mathrm{U}(m) \times \operatorname{Spin}(r)}{\left\{ \pm\left(\operatorname{Id}_{m}, 1\right), \pm\left(i \mathrm{Id}_{m},-\mathrm{vol}_{r}\right)\right\}}$ \\
\hline 3,5 & $\frac{\mathrm{Sp}(m) \times \operatorname{Spin}_{2}(r)}{\left\{ \pm\left(\mathrm{Id}_{m}, 1\right)\right\}}$ \\
\hline
\end{tabular}

- For $r \equiv 0(\bmod 8)$

\begin{tabular}{|c|c|c|c|}
\hline$m_{1}$ & 0 & $0(\bmod 2)$ & $1(\bmod 2)$ \\
\hline 0 & & $\frac{\mathrm{SO}\left(m_{2}\right) \times \operatorname{Spin}(r)}{\left\{ \pm\left(\mathrm{Id}_{m_{2}}, 1\right), \pm\left(\mathrm{Id}_{m_{2}},-\mathrm{vol}_{r}\right)\right\}}$ & $\frac{\mathrm{SO}\left(m_{2}\right) \times \operatorname{Spin}(r)}{\left\langle\left(\mathrm{Id}_{m_{2}},-\operatorname{vol}_{r}\right)\right\rangle}$ \\
\hline $0(\bmod 2)$ & $\frac{\mathrm{SO}\left(m_{1}\right) \times \operatorname{Spin}(r)}{\left\{ \pm\left(\mathrm{Id}_{m_{1}}, 1\right), \pm\left(\mathrm{Id}_{m_{1}}, \mathrm{vol}_{r}\right)\right\}}$ & $\frac{\mathrm{SO}\left(m_{1}\right) \times \mathrm{SO}\left(m_{2}\right) \times \operatorname{Spin}(r)}{\left\{ \pm\left(\mathrm{Id}_{m_{1}}, \mathrm{Id}_{m_{2}}, 1\right), \pm\left(\mathrm{Id}_{m_{1}},-\mathrm{Id}_{m_{2}}, \mathrm{vol}_{r}\right)\right\}}$ & $\frac{\mathrm{SO}\left(m_{1}\right) \times \mathrm{SO}\left(m_{2}\right) \times \operatorname{Spin}(r)}{\left\langle\left(-\mathrm{Id}_{m_{1}}, \mathrm{Id}_{m_{2}},-\operatorname{vol}_{r}\right)\right\rangle}$ \\
\hline $1(\bmod 2)$ & $\frac{\mathrm{SO}\left(m_{1}\right) \times \operatorname{Spin}_{2}(r)}{\left\langle\left(\mathrm{Id}_{m_{1}}, \mathrm{vol}_{r}\right)\right\rangle}$ & $\frac{\mathrm{SO}\left(m_{1}\right) \times \mathrm{SO}\left(m_{2}\right) \times \operatorname{Spin}(r)}{\left\langle\left(\mathrm{Id}_{m_{1}},-\mathrm{Id}_{m_{2}}, \mathrm{vol}_{r}\right)\right\rangle}$ & $\mathrm{SO}\left(m_{1}\right) \times \mathrm{SO}\left(m_{2}\right) \times \operatorname{Spin}(r)$ \\
\hline
\end{tabular}

- For $r \equiv 4(\bmod 8)$

\begin{tabular}{|c|c|c|c|}
\hline & $m_{1}, m_{2}>0$ & $m_{1}>0, m_{2}=0$ & $m_{1}=0, m_{2}>0$ \\
\hline$r=4$ & \multirow{2}{*}{$\frac{\operatorname{Sp}\left(m_{1}\right) \times \operatorname{Sp}\left(m_{2}\right) \times \operatorname{Spin}(r)}{\left\{ \pm\left(\operatorname{Id}_{2 m_{1}}, \operatorname{Id}_{2 m_{2}}, 1\right), \pm\left(\operatorname{Id}_{2 m_{1}},-\operatorname{Id}_{2 m_{2}}, \operatorname{vol}_{r}\right)\right\}}$} & $\frac{\operatorname{Sp}\left(m_{1}\right) \times \operatorname{Sppin}(3)}{\left\{ \pm\left(\operatorname{Id}_{2 m_{1}}, 1\right)\right\}}$ & $\frac{\operatorname{Sp}\left(m_{2}\right) \times \operatorname{Spin}(3)}{\left\{ \pm\left(\operatorname{Id}_{2 m_{2}}, 1\right)\right\}}$ \\
\hline$r>4$ & & $\frac{\operatorname{Sp}\left(m_{1}\right) \times \operatorname{Spin}(r)}{\left\{ \pm\left(\operatorname{Id}_{2 m_{1}}, 1\right), \pm\left(\operatorname{Id}_{2 m_{1}}, \mathrm{vol}_{r}\right)\right\}}$ & $\frac{\operatorname{Sp}\left(m_{2}\right) \times \operatorname{Spin}(r)}{\left\{ \pm\left(\operatorname{Id}_{2 m_{2}}, 1\right), \pm\left(\operatorname{Id}_{2 m_{2}},-\operatorname{vol}_{r}\right)\right\}}$ \\
\hline
\end{tabular}

Note that for $r=2$, the structure group is actually $\mathrm{U}(m)$.

Since all of these groups are quotients of products $G \times \operatorname{Spin}(r)$, where $G$ is a (product of) classical Lie group(s), it will be useful to know if they can be mapped to either $\operatorname{Spin}(r)$, or $\mathrm{SO}(r)$ or $\mathbb{P S O}(r)$. It is easy to see that they map as follows

- For $r \not \equiv 0(\bmod 4)$

\begin{tabular}{|c|c|c|}
\hline$r(\bmod 8)$ & 0 & 1 \\
\hline 1,7 & $\mathrm{SO}(r)$ & $\operatorname{Spin}(r)$ \\
\hline 2,6 & \multicolumn{2}{|c|}{$\operatorname{PSO}(r)$} \\
\hline 3,5 & \multicolumn{2}{|c|}{$\mathrm{SO}(r)$} \\
\hline
\end{tabular}

- For $r \equiv 0(\bmod 8)$

\begin{tabular}{|c|c|c|}
\hline$m_{1}$ & $0(\bmod 2)$ & $1(\bmod 2)$ \\
\hline $0(\bmod 2)$ & $\mathbb{P S O}(r)$ & $\operatorname{Spin}_{-}(r)$ \\
\hline $1(\bmod 2)$ & $\operatorname{Spin}_{+}(r)$ & $\operatorname{Spin}(r)$ \\
\hline
\end{tabular}

- For $r \equiv 4(\bmod 8)$

\begin{tabular}{|c|c|c|c|}
\hline$r \equiv 4(\bmod 8) \quad m_{1}, m_{2}$ & $m_{1}, m_{2}>0$ & $m_{1}>0, m_{2}=0$ & $m_{1}=0, m_{2}>0$ \\
\hline$r=4$ & \multirow{2}{*}{$\mathbb{P S O}(r)$} & $\mathrm{SO}(3)$ & $\mathrm{SO}(3)$ \\
\hline$r>4$ & & $\mathbb{P S O}(r)$ & $\mathbb{P S O}(r)$ \\
\hline
\end{tabular}


This can be summarized roughly as follows: the structure group of an almost- $\mathrm{Cl}_{r}^{0}$-Hermitian manifold of rank $r$ maps to $\mathrm{SO}(r)$ if $r$ is odd, and maps to $\mathbb{P S O}(r)$ if $r$ is even.

For future use, we will establish the notation for the decomposition of the complexified tangent bundle of an almost- $\mathrm{Cl}_{r}^{0}$-Hermitian manifold:

\begin{tabular}{|c|c|}
\hline$r(\bmod 8)$ & $T M \otimes \mathbb{C}$ \\
\hline 0 & $E_{1} \otimes \Delta_{r}^{+} \oplus E_{2} \otimes \Delta_{r}^{-}$ \\
\hline 1,7 & $E \otimes \Delta_{r}$ \\
\hline 2 & $E \otimes \Delta_{r}^{+} \oplus \bar{E} \otimes \Delta_{r}^{-}$ \\
\hline 6 & $\bar{E} \otimes \Delta_{r}^{+} \oplus E \otimes \Delta_{r}^{-}$ \\
\hline 3,5 & $E \otimes \Delta_{r}$ \\
\hline 4 & $E_{2} \otimes \Delta_{r}^{+} \oplus E_{1} \otimes \Delta_{r}^{-}$ \\
\hline
\end{tabular}

where $E, E_{1}, E_{2}$ are locally defined vector bundles with fibre $\mathbb{C}^{p}$ which correspond to the standard complex representation of the different Lie groups mentioned in (2.3).

\subsection{A useful lemma}

Lemma 2.5. Let $x \in \mathbb{C}$ and $k, m \in \mathbb{Z} / 2$ such that $k+m \in \mathbb{Z}$. If $|k|<|m|$, then

$$
G(z)=\frac{z^{k}}{z^{-m} e^{x}-z^{m} e^{-x}}
$$

is a rational function on $\mathbb{C}$ and

$$
\lim _{z \rightarrow 0} G(z)=0=\lim _{z \rightarrow \infty} G(z) .
$$

\section{Twisted spinor bundles on almost- $\mathrm{Cl}_{r}^{0}$-Hermitian manifolds}

In this subsection, we present some calculations relevant to the global definiton of twisted spinor bundles.

When the structure group of an oriented $N$-dimensional Riemannian manifold reduces to a proper subgroup $G \subset \mathrm{SO}(N)$, one can associate vector bundles to the corresponding $G$ principal bundle $P_{G}$ by means of the representations of $G$. If the manifold is Spin, one can ask if there exists a lifting map $\tilde{i}$ making the following diagram commute

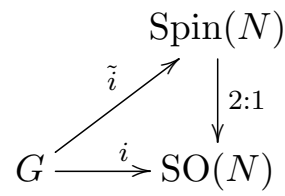

in which case, the Spin representation $\Delta_{N}$ may decompose according to $G$.

Even when such map $\tilde{i}$ does not exist (necessarily $\pi_{1}(G) \neq\{1\}$ ), there may be a finite covering space $G^{\prime}$ of $G=G^{\prime} / \Gamma$ for which it does, and one can then decompose the Spin representation according to $G^{\prime}$. We can now check how the elements of the finite subgroup $\Gamma$ act on $\Delta_{N}$, and at least some of them will act non-trivially, thus confirming that there cannot be a map $\tilde{i}$. By observing this action, we can then consider tensoring $\Delta_{N}$ with another representation $V$ of $G^{\prime}$ such that $\Gamma$ now acts trivially on $\Delta_{N} \otimes V$.

In the context of almost- $\mathrm{Cl}_{r}^{0}$-Hermitian manifolds, the structure group embeds into the relevant Spin group [1, Theorem 4.1], with the exception of four cases which we will analyze. More precisely, we found that 
- $\frac{\operatorname{Sp}(m) \times \operatorname{Spin}(3)}{\left\{ \pm\left(\operatorname{Id}_{2 m}, 1\right)\right\}}$ does not embed into $\operatorname{Spin}(4 m)$ if $m$ is odd;

- $\frac{\operatorname{Sp}\left(m_{1}\right) \times \operatorname{Sp}\left(m_{2}\right) \times \operatorname{Spin}(4)}{\left\{ \pm\left(\operatorname{Id}_{2 m_{1}}, \operatorname{Id}_{2 m_{2}}, 1\right), \pm\left(\operatorname{Id}_{2 m_{1}},-\operatorname{Id}_{2 m_{2}}, \operatorname{vol}_{4}\right)\right\}}$ does not embed into $\operatorname{Spin}\left(4\left(m_{1}+m_{2}\right)\right)$ if either $m_{1}$ or $m_{2}$ (or both) are odd;

- $\frac{\mathrm{U}(m) \times \operatorname{Spin}(6)}{\left\{ \pm\left(\operatorname{Id}_{m}, 1\right),\left(i \mathrm{Id}_{m},- \text { vol }_{6}\right)\right\}}$ does not embed into $\operatorname{Spin}(8 m)$ if $m$ is odd;

- $\frac{\mathrm{SO}\left(m_{1}\right) \times \mathrm{SO}\left(m_{2}\right) \times \operatorname{Spin}(8)}{\left\{\left(\operatorname{Id}_{m_{1}}, \mathrm{Id}_{m_{2}}, 1\right),\left(\operatorname{Id}_{m_{1}},-\mathrm{Id}_{2 m_{2}}, \operatorname{vol}_{8}\right)\right\}}$ if $m_{1}+1 \equiv m_{2} \equiv 0(\bmod 2), \frac{\mathrm{SO}\left(m_{1}\right) \times \operatorname{SO}\left(m_{2}\right) \times \operatorname{Spin}(8)}{\left\{\left(\operatorname{Id}_{m_{1}}, \mathrm{Id}_{m_{2}}, 1\right),\left(-\operatorname{Id}_{m_{1}}, \mathrm{Id}_{2 m_{2}},-\mathrm{vol}_{8}\right)\right\}}$ if $m_{1} \equiv m_{2}+1 \equiv 0(\bmod 2)$ do not embed into $\operatorname{Spin}\left(8\left(m_{1}+m_{2}\right)\right)$.

However, by the same calculations in [1] we know that there are homomorphisms

- $\operatorname{Sp}(m) \times \operatorname{Spin}(3) \longrightarrow \operatorname{Spin}(4 m)$;

- $\operatorname{Sp}\left(m_{1}\right) \times \operatorname{Sp}\left(m_{2}\right) \times \operatorname{Spin}(4) \longrightarrow \operatorname{Spin}\left(4\left(m_{1}+m_{2}\right)\right)$;

- $\mathrm{U}(m) \times \operatorname{Spin}(6) \longrightarrow \operatorname{Spin}(8 m)$;

- $\mathrm{SO}\left(m_{1}\right) \times \mathrm{SO}\left(m_{2}\right) \times \operatorname{Spin}(8) \longrightarrow \operatorname{Spin}\left(8\left(m_{1}+m_{2}\right)\right)$.

In order to analyze this situation and the appropriate twisting bundles for almost- $\mathrm{Cl}_{r}^{0}$ Hermitian manifolds in general, we need to set up some notation regarding weights of Lie groups.

\subsection{Weights of $\mathrm{SO}(N)$ with respect to the structure subgroups}

We need to rewrite the weights of $\mathrm{SO}(N)$ in terms of the maximal torus of the relevant structure group. Let $\left(\eta_{1}, \ldots, \eta_{N / 2}\right)$ denote the coordinates of a maximal torus of $\mathrm{SO}(N)$, and $\left(\varphi_{1}, \ldots, \varphi_{\left[\frac{r}{2}\right]}\right)$ denote the coordinates of a maximal torus of $\mathrm{SO}(r)$.

For $r$ odd, let $\left.\lambda_{1}, \ldots, \lambda_{2} \frac{r}{2}\right]$ denote the weights of $\Delta_{r}$

$$
\pm \frac{1}{2} \varphi_{1} \pm \cdots \pm \frac{1}{2} \varphi_{\left[\frac{r}{2}\right]}
$$

listed in some order such that the first half of weights have an even number of negative signs, and the second half of weights have an odd number of negative signs.

For $r$ even, let $\lambda_{1}^{ \pm}, \ldots, \lambda_{2^{\frac{r}{2}-1}}^{ \pm}$denote the weights of $\Delta_{r}^{ \pm}$

$$
\pm \frac{1}{2} \varphi_{1} \pm \cdots \pm \frac{1}{2} \varphi_{\frac{r}{2}}
$$

which have an even and odd number of negative signs respectively. If $r \equiv 0 \bmod 4$ we will be considering $\lambda_{1}^{ \pm}, \ldots, \lambda_{2^{\frac{r}{2}-1}}^{ \pm}$to be listed in some order so that the first and second halves are interchanged by reflection (changing all the signs),

\subsection{1 $r \equiv 1,7 \bmod 8$}

Let $\left(\theta_{1}, \ldots, \theta_{\left[\frac{m}{2}\right]}\right)$ denote the coordinates of maximal tori of $\mathrm{SO}(m)$. Since

$$
\mathbb{C}^{N}=\mathbb{C}^{m} \otimes \Delta_{r}
$$

we can set

$$
\eta_{(j-1) 2^{\left[\frac{r}{2}\right]}+k}=\theta_{j}+\lambda_{k}
$$

if $m$ is even, and

$$
\eta_{(j-1) 2^{\left[\frac{r}{2}\right]}+k}=\theta_{j}+\lambda_{k}, \quad \eta_{\left[\frac{m}{2}\right] 2^{\left[\frac{r}{2}\right]}+l}=\lambda_{l}
$$

if $m$ is odd, where $1 \leq j \leq\left[\frac{m}{2}\right], 1 \leq k \leq 2^{\left[\frac{r}{2}\right]}$ and $1 \leq l \leq 2^{\left[\frac{r}{2}\right]-1}$ in both cases. 


\subsection{2 $r \equiv 2,6 \bmod 8$}

Let $\left(\theta_{1}, \ldots, \theta_{m}\right)$ denote the coordinates of maximal tori of $\mathrm{U}(m)$. Since

$$
\mathbb{C}^{N}= \begin{cases}\mathbb{C}^{m} \otimes \Delta_{r}^{+} \oplus \overline{\mathbb{C}^{m}} \otimes \Delta_{r}^{-} & \text {if } r \equiv 2(\bmod 8), \\ \overline{\mathbb{C}^{m}} \otimes \Delta_{r}^{+} \oplus \mathbb{C}^{m} \otimes \Delta_{r}^{-} & \text {if } r \equiv 6(\bmod 8),\end{cases}
$$

we can set

$$
\eta_{(j-1) 2^{\frac{r}{2}-1}+k}= \begin{cases}\theta_{j}+\lambda_{k}^{+} & \text {if } r \equiv 2(\bmod 8), \\ \theta_{j}+\lambda_{k}^{-} & \text {if } r \equiv 6(\bmod 8),\end{cases}
$$

where $1 \leq j \leq m$ and $1 \leq k \leq 2^{\frac{r}{2}-1}$.

\subsection{3 $r \equiv 3,5 \bmod 8$}

Let $\left(\theta_{1}, \ldots, \theta_{m}\right)$ denote the coordinates of maximal tori of $\operatorname{Sp}(m)$. Since

$$
\mathbb{C}^{N}=\mathbb{C}^{2 m} \otimes \Delta_{r}
$$

we can set

$$
\eta_{(j-1) 2^{\left[\frac{r}{2}\right]}+k}=\theta_{j}+\lambda_{k}
$$

where $1 \leq j \leq m$ and $1 \leq k \leq 2^{\left[\frac{r}{2}\right]}$.

\subsection{4 $r \equiv 4 \bmod 8$}

Let $\left(\theta_{1}, \ldots, \theta_{m_{1}}\right)$ and $\left(\theta_{1}^{\prime}, \ldots, \theta_{m_{2}}^{\prime}\right)$ denote the coordinates of maximal tori of $\operatorname{Sp}\left(m_{1}\right)$ and $\operatorname{Sp}\left(m_{2}\right)$ respectively. Since

$$
\mathbb{C}^{N}=\mathbb{C}^{2 m_{1}} \otimes \Delta_{r}^{+} \oplus \mathbb{C}^{2 m_{2}} \otimes \Delta_{r}^{-},
$$

we can set

$$
\eta_{\left(j_{1}-1\right) 2^{\frac{r}{2}-1}+k}=\theta_{j_{1}}+\lambda_{k}^{+}, \quad \eta_{m_{1} 2^{\frac{r}{2}-1}+\left(j_{2}-1\right) 2^{\frac{r}{2}-1}+k}=\theta_{j_{2}}^{\prime}+\lambda_{k}^{-},
$$

where $1 \leq j_{1} \leq m_{1}, 1 \leq j_{2} \leq m_{2}$ and $1 \leq k \leq 2^{\frac{r}{2}-1}$.

\subsection{5 $r \equiv 0 \bmod 8$}

Let $\left(\theta_{1}, \ldots, \theta_{\left[\frac{m_{1}}{2}\right]}\right)$ and $\left(\theta_{1}^{\prime}, \ldots, \theta_{\left[\frac{m_{2}}{2}\right]}^{\prime}\right)$ denote the coordinates of maximal tori of $\mathrm{SO}\left(m_{1}\right)$ and $\mathrm{SO}\left(m_{2}\right)$ respectively. Since

$$
\mathbb{C}^{N}=\mathbb{C}^{m_{1}} \otimes \Delta_{r}^{+} \oplus \mathbb{C}^{m_{2}} \otimes \Delta_{r}^{-},
$$

we can set

- if $m_{1}, m_{2}$ are even,

$$
\eta_{\left(j_{1}-1\right) 2^{\frac{r}{2}-1}+k}=\theta_{j_{1}}+\lambda_{k}^{+}, \quad \eta_{m_{1} 2^{\frac{r}{2}-1}+\left(j_{2}-1\right) 2^{\frac{r}{2}-1}+k}=\theta_{j_{2}}^{\prime}+\lambda_{k}^{-},
$$

where $1 \leq j_{1} \leq \frac{m_{1}}{2}, 1 \leq j_{2} \leq \frac{m_{2}}{2}$ and $1 \leq k \leq 2^{\frac{r}{2}-1}$; 
- if $m_{1}$ is even and $m_{2}$ is odd,

$$
\begin{aligned}
& \eta_{\left(j_{1}-1\right) 2^{\frac{r}{2}-1}+k}=\theta_{j_{1}}+\lambda_{k}^{+}, \quad \eta_{m_{1} 2^{\frac{r}{2}-1}+\left(j_{2}-1\right) 2^{\frac{r}{2}-1}+k}=\theta_{j_{2}}^{\prime}+\lambda_{k}^{-}, \\
& \eta_{m_{1} 2^{\frac{r}{2}-1}+\left[\frac{m_{2}}{2}\right] 2^{\frac{r}{2}-1}+l}=\lambda_{l}^{-},
\end{aligned}
$$

where $1 \leq j_{1} \leq \frac{m_{1}}{2}, 1 \leq j_{2} \leq\left[\frac{m_{2}}{2}\right], 1 \leq k \leq 2^{\frac{r}{2}-1}$ and $1 \leq l \leq 2^{\frac{r}{2}-2}$;

- if $m_{1}$ is odd and $m_{2}$ is even,

$$
\begin{aligned}
& \eta_{\left(j_{1}-1\right) 2^{\frac{r}{2}-1}+k}=\theta_{j_{1}}+\lambda_{k}^{+}, \quad \eta_{\left[\frac{m_{1}}{2}\right] 2^{\frac{r}{2}-1}+l}=\lambda_{l}^{+}, \\
& \eta_{\left[\frac{m_{1}}{2}\right] 2^{\frac{r}{2}-1}+2^{\frac{r}{2}-2}+\left(j_{2}-1\right) 2^{\frac{r}{2}-1}+k}=\theta_{j_{2}}^{\prime}+\lambda_{k}^{-},
\end{aligned}
$$

where $1 \leq j_{1} \leq\left[\frac{m_{1}}{2}\right], 1 \leq j_{2} \leq \frac{m_{2}}{2}, 1 \leq k \leq 2^{\frac{r}{2}-1}$ and $1 \leq l \leq 2^{\frac{r}{2}-2}$;

- if $m_{1}, m_{2}$ are odd,

$$
\begin{aligned}
& \eta_{\left(j_{1}-1\right) 2^{\frac{r}{2}-1}+k}=\theta_{j_{1}}+\lambda_{k}^{+}, \quad \eta_{\left[\frac{m_{1}}{2}\right] 2^{\frac{r}{2}-1}+l}=\lambda_{l}^{+}, \\
& \eta_{\left[\frac{m_{1}}{2}\right] 2^{\frac{r}{2}-1}+2^{\frac{r}{2}-2}+\left(j_{2}-1\right) 2^{\frac{r}{2}-1}+k}=\theta_{j_{2}}^{\prime}+\lambda_{k}^{-}, \quad \eta_{\left[\frac{m_{1}}{2}\right] 2^{\frac{r}{2}-1}+2^{\frac{r}{2}-2}+\left[\frac{m_{2}}{2}\right] 2^{\frac{r}{2}-1}+l}=\lambda_{l}^{-},
\end{aligned}
$$

where $1 \leq j_{1} \leq\left[\frac{m_{1}}{2}\right], 1 \leq j_{2} \leq\left[\frac{m_{2}}{2}\right], 1 \leq k \leq 2^{\frac{r}{2}-1}$ and $1 \leq l \leq 2^{\frac{r}{2}-2}$.

\subsection{The Spin representation when $r=3,4,6,8$}

The elements of the finite subgroups involved in the structure groups of almost- $\mathrm{Cl}_{r}^{0}$-Hermitian manifolds actually belong to maximal tori. Thus we can calculate their effect on representations in terms of the weights we just described. In this subsection, we examine the cases when the structure group does not embed into $\operatorname{Spin}(N)$.

\subsection{1 $r=3$}

Recall (3.2), which in this case is

$$
\eta_{2 j-1}=\theta_{j}+\frac{\varphi_{1}}{2}, \quad \eta_{2 j}=\theta_{j}-\frac{\varphi_{1}}{2},
$$

so that the weights of the spin representation are

$$
\pm \frac{\eta_{1}}{2} \pm \cdots \pm \frac{\eta_{2 m}}{2}=\sum_{j \in I_{1}} \frac{\theta_{j}+\frac{\varphi_{1}}{2}}{2}-\sum_{j \in \bar{I}_{1}} \frac{\theta_{j}+\frac{\varphi_{1}}{2}}{2}+\sum_{j \in I_{2}} \frac{\theta_{j}-\frac{\varphi_{1}}{2}}{2}-\sum_{j \in \bar{I}_{2}} \frac{\theta_{j}-\frac{\varphi_{1}}{2}}{2}
$$

where $I_{1}, I_{2} \subseteq\{1, \ldots, m\}$, and $\bar{I}_{j}=\{1, \ldots, m\}-I_{j}$ denote their complements, $j=1,2$. The element $\left(-\operatorname{Id}_{2 m},-1\right) \in \operatorname{Sp}(m) \times \operatorname{Spin}(3)$ corresponds to the parameters

$$
\theta_{j}=\pi, \quad \varphi_{1}=2 \pi,
$$

for $1 \leq j \leq m$, so that such a sum is equal to

$$
2\left|I_{1}\right| \pi-m \pi
$$

and the effect of $\left(-\mathrm{Id}_{2 m},-1\right)$ on each weight line is

$$
e^{-i m \pi}=(-1)^{m} \text {. }
$$

Thus, $\left(-\operatorname{Id}_{2 m},-1\right) \in \operatorname{Sp}(m) \times \operatorname{Spin}(3)$ acts trivially on $\Delta_{4 m}$ if $m$ is even and as multiplication by $(-1)$ if $m$ is odd.

Thus, in order to have a twisted Spin representation

$$
\Delta_{4 m} \otimes \bigwedge^{u} \mathbb{C}^{2 m} \otimes\left(\Delta_{3}\right)^{\otimes s}
$$

of $\frac{\operatorname{Sp}(m) \times \operatorname{Spin}(3)}{\left\{ \pm\left(\operatorname{Id}_{2 m}, 1\right)\right\}}$, the exponents must satisfy $m+u+s \equiv 0(\bmod 2)$, which is a well known fact for almost quaternion-Hermitian manifolds [21]. 


\subsection{2 $r=4$}

By (3.3), the weights of the spin representation are

$$
\begin{aligned}
\pm & \frac{\eta_{1}}{2} \pm \cdots \pm \frac{\eta_{2 m_{1}+2 m_{2}}}{2} \\
= & \sum_{j_{1} \in I_{1}} \frac{\theta_{j_{1}}+\frac{\varphi_{1}+\varphi_{2}}{2}}{2}-\sum_{j_{1} \in \bar{I}_{1}} \frac{\theta_{j_{1}}+\frac{\varphi_{1}+\varphi_{2}}{2}}{2}+\sum_{j_{1} \in I_{2}} \frac{\theta_{j_{1}}+\frac{-\varphi_{1}-\varphi_{2}}{2}}{2}-\sum_{j_{1} \in \bar{I}_{2}} \frac{\theta_{j_{1}}+\frac{-\varphi_{1}-\varphi_{2}}{2}}{2} \\
& +\sum_{j_{2} \in I_{1}^{\prime}} \frac{\theta_{j_{2}}^{\prime}+\frac{-\varphi_{1}+\varphi_{2}}{2}}{2}-\sum_{j_{2} \in \bar{I}_{1}^{\prime}} \frac{\theta_{j_{2}}^{\prime}+\frac{-\varphi_{1}+\varphi_{2}}{2}}{2}+\sum_{j_{2} \in I_{2}^{\prime}} \frac{\theta_{j_{2}}^{\prime}+\frac{\varphi_{1}-\varphi_{2}}{2}}{2}-\sum_{j_{2} \in \bar{I}_{2}^{\prime}} \frac{\theta_{j_{2}}^{\prime}+\frac{\varphi_{1}-\varphi_{2}}{2}}{2},
\end{aligned}
$$

where $I_{1}, I_{2} \subseteq\left\{1, \ldots, m_{1}\right\}$ and $I_{1}^{\prime}, I_{2}^{\prime} \subseteq\left\{1, \ldots, m_{2}\right\}$.

- The element $\left(-\mathrm{Id}_{2 m_{1}},-\mathrm{Id}_{2 m_{2}},-1\right) \in \operatorname{Sp}\left(m_{1}\right) \times \operatorname{Sp}\left(m_{2}\right) \times \operatorname{Spin}(4)$ corresponds to the parameters

$$
\theta_{j_{1}}=\pi, \quad \theta_{j_{2}}^{\prime}=\pi, \quad \varphi_{1}=2 \pi, \quad \varphi_{2}=0,
$$

so that such a sum is equal to

$$
\pi\left(2\left|I_{1}\right|-m_{1}+2\left|I_{2}^{\prime}\right|-m_{2}\right)
$$

and the effect of $\left(-\mathrm{Id}_{2 m_{1}},-\mathrm{Id}_{2 m_{2}},-1\right)$ on each weight line is

$$
e^{-i \pi\left(m_{1}+m_{2}\right)}=(-1)^{m_{1}+m_{2}} \text {. }
$$

- The element $\left(\operatorname{Id}_{2 m_{1}},-\mathrm{Id}_{2 m_{2}}, \operatorname{vol}_{4}\right) \in \operatorname{Sp}\left(m_{1}\right) \times \operatorname{Sp}\left(m_{2}\right) \times \operatorname{Spin}(4)$ corresponds to the parameters

$$
\theta_{j_{1}}=0, \quad \theta_{j_{2}}^{\prime}=\pi, \quad \varphi_{1}=\pi, \quad \varphi_{2}=\pi,
$$

so that the effect of $\left(\operatorname{Id}_{2 m_{1}},-\mathrm{Id}_{2 m_{2}}, \mathrm{vol}_{4}\right)$ on each weight line is

$$
e^{-i \pi m_{2}}=(-1)^{m_{2}}
$$

- The element $\left(-\mathrm{Id}_{2 m_{1}}, \mathrm{Id}_{2 m_{2}},-\operatorname{vol}_{4}\right) \in \operatorname{Sp}\left(m_{1}\right) \times \operatorname{Sp}\left(m_{2}\right) \times \operatorname{Spin}(4)$ corresponds to the parameters

$$
\theta_{j_{1}}=\pi, \quad \theta_{j_{2}}^{\prime}=0, \quad \varphi_{1}=\pi, \quad \varphi_{2}=-\pi,
$$

so that the effect of $\left(-\mathrm{Id}_{2 m_{1}}, \mathrm{Id}_{2 m_{2}},-\mathrm{vol}_{4}\right)$ on each weight line is

$$
e^{-i \pi m_{1}}=(-1)^{m_{1}}
$$

Thus, in order to have a twisted Spin representation

$$
\Delta_{4\left(m_{1}+m_{2}\right)} \otimes \bigwedge^{u_{1}} \mathbb{C}^{2 m_{1}} \otimes \bigwedge^{u_{2}} \mathbb{C}^{2 m_{2}} \otimes\left(\Delta_{4}^{+}\right)^{\otimes s} \otimes\left(\Delta_{4}^{-}\right)^{\otimes t}
$$

of

$$
\frac{\operatorname{Sp}\left(m_{1}\right) \times \operatorname{Sp}\left(m_{2}\right) \times \operatorname{Spin}(4)}{\left\{ \pm\left(\operatorname{Id}_{2 m_{1}}, \operatorname{Id}_{2 m_{2}}, 1\right), \pm\left(\operatorname{Id}_{2 m_{1}},-\mathrm{Id}_{2 m_{2}}, \operatorname{vol}_{4}\right)\right\}}
$$

the exponents must satisfy

$$
m_{1}+u_{1}+t \equiv 0(\bmod 2), \quad m_{2}+u_{2}+s \equiv 0(\bmod 2) .
$$




\subsection{3 $r=6$}

By (3.1), the weights of the spin representation are

$$
\begin{aligned}
& \pm \frac{\eta_{1}}{2} \pm \cdots \pm \frac{\eta_{8 m}}{2} \\
& =\sum_{j \in I_{1}} \frac{\theta_{j}+\frac{-\varphi_{1}+\varphi_{2}+\varphi_{3}}{2}}{2}-\sum_{j \in \bar{I}_{1}} \frac{\theta_{j}+\frac{-\varphi_{1}+\varphi_{2}+\varphi_{3}}{2}}{2}+\sum_{j \in I_{2}} \frac{\theta_{j}+\frac{\varphi_{1}-\varphi_{2}+\varphi_{3}}{2}}{2}-\sum_{j \in \bar{I}_{2}} \frac{\theta_{j}+\frac{\varphi_{1}-\varphi_{2}+\varphi_{3}}{2}}{2} \\
& +\sum_{j \in I_{3}} \frac{\theta_{j}+\frac{\varphi_{1}+\varphi_{2}-\varphi_{3}}{2}}{2}-\sum_{j \in \overline{I_{3}}} \frac{\theta_{j}+\frac{\varphi_{1}+\varphi_{2}-\varphi_{3}}{2}}{2}+\sum_{j \in I_{4}} \frac{\theta_{j}+\frac{-\varphi_{1}-\varphi_{2}-\varphi_{3}}{2}}{2}-\sum_{j \in \bar{I}_{4}} \frac{\theta_{j}+\frac{-\varphi_{1}-\varphi_{2}-\varphi_{3}}{2}}{2},
\end{aligned}
$$

where $I_{1}, I_{2}, I_{3}, I_{4} \subseteq\left\{1, \ldots, m_{1}\right\}$.

- The element $\left(-\mathrm{Id}_{m},-1\right) \in \mathrm{U}(m) \times \operatorname{Spin}(6)$ corresponds to the parameters

$$
\theta_{j}=\pi, \quad \varphi_{1}=2 \pi, \quad \varphi_{2}=0, \quad \varphi_{3}=0,
$$

so that its effect on each weight line is

$$
e^{-2 i \pi m}=1
$$

- The element $\left(i \mathrm{Id}_{m},-\mathrm{vol}_{6}\right) \in \mathrm{U}(m) \times \operatorname{Spin}(6)$ corresponds to the parameters

$$
\theta_{j}=\frac{\pi}{2}, \quad \varphi_{1}=-\pi, \quad \varphi_{2}=\pi, \quad \varphi_{3}=\pi,
$$

so that its effect on each weight line is

$$
e^{-i \pi m}=(-1)^{m}
$$

Thus, in order to have a twisted Spin representation

$$
\Delta_{8 m} \otimes \bigwedge^{u_{1}} \mathbb{C}^{m} \otimes \bigwedge^{u_{2}} \overline{\mathbb{C}^{m}} \otimes\left(\Delta_{6}^{+}\right)^{\otimes s} \otimes\left(\Delta_{6}^{-}\right)^{\otimes t}
$$

of $\frac{\mathrm{U}(m) \times \operatorname{Spin}(6)}{\left\{ \pm\left(\operatorname{Id}_{m}, 1\right), \pm\left(i \mathrm{Id}_{m},-\mathrm{vol}_{6}\right)\right\}}$, the exponents must satisfy

$$
\begin{aligned}
& u_{1}+u_{2}+s+t \equiv 0(\bmod 2), \quad 2 m+u_{1}+3 u_{2}+s+3 t \equiv 0(\bmod 4), \\
& 2 m+3 u_{1}+u_{2}+3 s+t \equiv 0(\bmod 4)
\end{aligned}
$$

\subsection{4 $r=8$}

By (3.5), if $m_{1}+1 \equiv m_{2} \equiv 0(\bmod 2)$, the element $\left(\operatorname{Id}_{m_{1}},-\mathrm{Id}_{m_{2}}, \operatorname{vol}_{8}\right) \in \mathrm{SO}\left(m_{1}\right) \times \mathrm{SO}\left(m_{2}\right) \times$ $\operatorname{Spin}(8)$ corresponds to the parameters

$$
\theta_{j_{1}}=0, \quad \theta_{j_{2}}^{\prime}=\pi, \quad \varphi_{1}=\varphi_{2}=\varphi_{3}=\varphi_{4}=\pi,
$$

and its effect on each weight line is mutiplication by -1 . Thus, we can have twisted Spin representations

$$
\Delta_{8\left(m_{1}+m_{2}\right)} \otimes \bigwedge^{u_{1}} \mathbb{C}^{m_{1}} \otimes \bigwedge^{u_{2}} \mathbb{C}^{m_{2}} \otimes\left(\Delta_{8}^{+}\right)^{\otimes s} \otimes\left(\Delta_{8}^{-}\right)^{\otimes t}
$$

of

$$
\frac{\mathrm{SO}\left(m_{1}\right) \times \mathrm{SO}\left(m_{2}\right) \times \operatorname{Spin}(8)}{\left\{\left(\mathrm{Id}_{m_{1}}, \mathrm{Id}_{m_{2}}, 1\right),\left(\mathrm{Id}_{m_{1}},-\mathrm{Id}_{m_{2}}, \operatorname{vol}_{8}\right)\right\}}
$$

if $u_{2}+t \equiv 1(\bmod 2)$ and $u_{1}, s \in \mathbb{N}$. 
Similarly, by $(3.4)$, if $m_{1} \equiv m_{2}+1 \equiv 0(\bmod 2)$, we can have twisted Spin representations

$$
\Delta_{8\left(m_{1}+m_{2}\right)} \otimes \bigwedge^{u_{1}} \mathbb{C}^{m_{1}} \otimes \bigwedge^{u_{2}} \mathbb{C}^{m_{2}} \otimes\left(\Delta_{8}^{+}\right)^{\otimes s} \otimes\left(\Delta_{8}^{-}\right)^{\otimes t}
$$

of

$$
\frac{\mathrm{SO}\left(m_{1}\right) \times \mathrm{SO}\left(m_{2}\right) \times \operatorname{Spin}(8)}{\left\{\left(\mathrm{Id}_{m_{1}}, \mathrm{Id}_{m_{2}}, 1\right),\left(-\mathrm{Id}_{m_{1}}, \operatorname{Id}_{m_{2}},-\operatorname{vol}_{8}\right)\right\}}
$$

if $u_{1}+s \equiv 1(\bmod 2)$ and $u_{2}, t \in \mathbb{N}$.

\subsection{Twisting representations}

For most $r$, almost-Cl ${ }_{r}^{0}$-Hermitian manifolds are Spin [1, Theorem 4.1]. In particular, this is the case when $r \geq 5$ and $r \neq 6,8$. Thus, we only need to choose suitable representations of the structure group $G$ to twist the spinor bundle:

- For $r \equiv 1,7(\bmod 8)$ our candidates are

$$
\bigwedge^{u} \mathbb{C}^{m} \otimes \Delta_{r}^{\otimes s}, \quad S^{u} \mathbb{C}^{m} \otimes \Delta_{r}^{\otimes s}
$$

They are representations of the structure group when

$$
\begin{array}{ll}
u+s \equiv 0(\bmod 2) & \text { if } m \text { is even, } \\
u, s \in \mathbb{N} & \text { if } m \text { is odd. }
\end{array}
$$

- For $r \equiv 3,5(\bmod 8)$ our candidates are

$$
\bigwedge^{u} \mathbb{C}^{2 m} \otimes \Delta_{r}^{\otimes s}, \quad S^{u} \mathbb{C}^{2 m} \otimes \Delta_{r}^{\otimes s} .
$$

They are representations of the structure group when

$$
u+s \equiv 0(\bmod 2)
$$

- For $r \equiv 4(\bmod 8)$ our candidates are

$$
\begin{aligned}
& \bigwedge^{u_{1}} \mathbb{C}^{2 m_{1}} \otimes \bigwedge^{u_{2}} \mathbb{C}^{2 m_{2}} \otimes\left(\Delta_{r}^{+}\right)^{\otimes s} \otimes\left(\Delta_{r}^{-}\right)^{\otimes t} \\
& S^{u_{1}} \mathbb{C}^{2 m_{1}} \otimes S^{u_{2}} \mathbb{C}^{2 m_{2}} \otimes\left(\Delta_{r}^{+}\right)^{\otimes s} \otimes\left(\Delta_{r}^{-}\right)^{\otimes t} .
\end{aligned}
$$

They are representations of the structure group when

$$
u_{2}+s \equiv 0(\bmod 2), \quad u_{1}+t \equiv 0(\bmod 2) .
$$

- For $r \equiv 2(\bmod 8), r \neq 2$, our candidates are

$$
\bigwedge^{u_{1}} \mathbb{C}^{m} \otimes \bigwedge^{u_{2}} \overline{\mathbb{C}^{m}} \otimes\left(\Delta_{r}^{+}\right)^{\otimes s} \otimes\left(\Delta_{r}^{-}\right)^{\otimes t}, \quad S^{u_{1}} \mathbb{C}^{m} \otimes S^{u_{2}} \overline{\mathbb{C}^{m}} \otimes\left(\Delta_{r}^{+}\right)^{\otimes s} \otimes\left(\Delta_{r}^{-}\right)^{\otimes t} .
$$

They are representations of the structure group when

$$
\begin{aligned}
& u_{1}+u_{2}+s+t \equiv 0(\bmod 2), \quad u_{1}+3 u_{2}+3 s+t \equiv 0(\bmod 4), \\
& 3 u_{1}+u_{2}+s+3 t \equiv 0(\bmod 4) .
\end{aligned}
$$


- For $r \equiv 6(\bmod 8)$ our candidates are

$$
\bigwedge^{u_{1}} \mathbb{C}^{m} \otimes \bigwedge^{u_{2}} \overline{\mathbb{C}^{m}} \otimes\left(\Delta_{r}^{+}\right)^{\otimes s} \otimes\left(\Delta_{r}^{-}\right)^{\otimes t}, \quad S^{u_{1}} \mathbb{C}^{m} \otimes S^{u_{2}} \overline{\mathbb{C}^{m}} \otimes\left(\Delta_{r}^{+}\right)^{\otimes s} \otimes\left(\Delta_{r}^{-}\right)^{\otimes t} .
$$

They are representations of the structure group when

$$
\begin{aligned}
& u_{1}+u_{2}+s+t \equiv 0(\bmod 2), \quad u_{1}+3 u_{2}+s+3 t \equiv 0(\bmod 4), \\
& 3 u_{1}+u_{2}+3 s+t \equiv 0(\bmod 4) .
\end{aligned}
$$

- For $r \equiv 0(\bmod 8)$ our candidates are

$$
\begin{aligned}
& \bigwedge^{u_{1}} \mathbb{C}^{m_{1}} \otimes \bigwedge^{u_{2}} \mathbb{C}^{m_{2}} \otimes\left(\Delta_{r}^{+}\right)^{\otimes s} \otimes\left(\Delta_{r}^{-}\right)^{\otimes t}, \\
& S^{u_{1}} \mathbb{C}^{m_{1}} \otimes S^{u_{2}} \mathbb{C}^{m_{2}} \otimes\left(\Delta_{r}^{+}\right)^{\otimes s} \otimes\left(\Delta_{r}^{-}\right)^{\otimes t} .
\end{aligned}
$$

They are representations of the structure group when

$$
\begin{aligned}
& \left\{\begin{array}{l}
u_{2}+t \equiv 0(\bmod 2) \\
u_{1}+s \equiv 0(\bmod 2)
\end{array} \quad \text { if } m_{1} \equiv m_{2} \equiv 0(\bmod 2),\right. \\
& \left\{\begin{array}{l}
u_{2}+t \equiv 0(\bmod 2) \\
u_{1}, s \in \mathbb{N}
\end{array} \quad \text { if } m_{1}+1 \equiv m_{2} \equiv 0(\bmod 2),\right. \\
& \left\{\begin{array}{l}
u_{2}, t \in \mathbb{N} \\
u_{1}+s \equiv 0(\bmod 2)
\end{array} \quad \text { if } m_{1} \equiv m_{2}+1 \equiv 0(\bmod 2),\right. \\
& u_{1}, u_{2}, s, t \in \mathbb{N} \quad \text { if } m_{1} \equiv m_{2} \equiv 0(\bmod 2) \text {. }
\end{aligned}
$$

\section{Index calculations}

In this section, we recall the definition of twisted Dirac operators, how to apply the AtiyahSinger fixed point formula [4], (infinitesimal) automorphisms of almost- $\mathrm{Cl}_{r}^{0}$-Hermitian manifolds and prove the vanishing Theorems 4.7, 4.8 and 4.9.

\subsection{Rigidity of elliptic operators}

Definition 4.1. Let $D: \Gamma(E) \longrightarrow \Gamma(F)$ be an elliptic operator acting on sections of the vector bundles $E$ and $F$ over a compact manifold $M$. The index of $D$ is the virtual vector space $\operatorname{ind}(D)=\operatorname{ker}(D)-\operatorname{coker}(D)$. If $M$ admits a circle action preserving $D$, i.e., such that $S^{1}$ acts on $E$ and $F$, and commutes with $D$, ind $(D)$ admits a Fourier decomposition into complex 1-dimensional irreducible representations of $S^{1} \operatorname{ind}(D)=\sum a_{m} L^{m}$, where $a_{m} \in \mathbb{Z}$ and $L^{m}$ is the representation of $S^{1}$ on $\mathbb{C}$ given by $\lambda \mapsto \lambda^{m}$. The elliptic operator $D$ is called rigid if $a_{m}=0$ for all $m \neq 0$, i.e., $\operatorname{ind}(D)$ consists only of the trivial representation with multiplicity $a_{0}$.

Let us recall three examples.

Example 4.2. The deRham complex

$$
d+d^{*}: \Omega^{\text {even }} \longrightarrow \Omega^{\text {odd }}
$$

from even-dimensional forms to odd-dimensional ones, where $d^{*}$ denotes the adjoint of the exterior derivative $d$, is rigid for any circle action on $M$ by isometries since by Hodge theory the kernel and the cokernel of this operator consist of harmonic forms, which by homotopy invariance stay fixed under the circle action. 
Example 4.3. The signature operator on an oriented manifold

$$
d_{s}: \Omega_{c}^{+} \longrightarrow \Omega_{c}^{-}
$$

from even to odd complex forms under the Hodge $*$ operator is rigid for any circle action on $M$ by isometries since the kernel and cokernel of this operator consist of harmonic forms.

Example 4.4. The Dirac operator on a Spin manifold is rigid for any circle action by isometries [3].

\subsection{Twisted Dirac operators}

In this subsection, let $M$ be a $4 n$-dimensional oriented Riemannian manifold. $M$ is Spin if its orthonormal frame bundle $P_{\mathrm{SO}(4 n)}$ admits a double cover by a principal bundle $P_{\mathrm{Spin}(4 n)}$ with structure group $\operatorname{Spin}(4 n)$, which gives rise to the spinor bundle

$$
P_{\operatorname{Spin}(4 n)} \times{ }_{\kappa} \Delta_{4 n}
$$

The Levi-Civita connection on $P_{\mathrm{SO}(4 n)}$ can be lifted to $P_{\mathrm{Spin}(4 n)}$ to define a covariant differentiation $\nabla$ on $\Delta$

$$
\nabla: \Gamma(\Delta) \longrightarrow \Gamma\left(T^{*} \otimes \Delta\right)
$$

and the (elliptic and self-adjoint) Dirac operator

$$
\not(\psi)=\sum_{i=1}^{4 n} e^{i} \cdot \nabla_{e_{i}} \psi
$$

for $\psi \in \Gamma(\Delta)$, where $\left(e_{1}, \ldots, e_{4 n}\right)$ is a local orthonormal frame. Since the spin representation decomposes, the Dirac operator can be split into two parts

$$
\not \partial: \Gamma\left(\Delta_{+}\right) \longrightarrow \Gamma\left(\Delta_{-}\right), \quad \not{ }^{*}: \Gamma\left(\Delta_{-}\right) \longrightarrow \Gamma\left(\Delta_{+}\right) .
$$

We are interested in Dirac operators with coefficients in auxiliary vector bundles $F$ equipped with a covariant derivative $\nabla^{F}: \Gamma(F) \longrightarrow \Gamma\left(T^{*} \otimes F\right)$. The Dirac operator twisted by $F$ (or with coefficients in $F$ )

$$
(\not \otimes F): \Gamma\left(\Delta_{+} \otimes F\right) \longrightarrow \Gamma\left(\Delta_{-} \otimes F\right)
$$

is defined by

$$
(\not \otimes F)(\psi \otimes f)=\left(\sum_{i=1}^{4 n} e^{i} \cdot \nabla_{e_{i}} \psi\right) \otimes f+\sum_{i=0}^{4 n} \mu\left(e^{i} \otimes \psi\right) \otimes \nabla_{e_{i}}^{F} f,
$$

where $\psi \in \Gamma(\Delta), f \in \Gamma(F)$.

Remark 4.5. If the manifold is not Spin, there may exist well defined twisted spinor bundles (as above), as it happens when the structure group of $M$ reduces to a subgroup of $\mathrm{SO}(4 n)$ and $\Delta_{4 n} \otimes F$ is a representation of such subgroup. 


\subsection{Index formula and localization}

Let $M$ be an compact $4 n$-dimensional oriented Riemannian manifold. Let us assume that the bundle

$$
\Delta_{4 n} \otimes F
$$

is well defined, where we will use the same symbol to denote the representation and the associated vector bundle, where the $\operatorname{dim}_{\mathbb{C}}(F)=p$. Since $\Delta_{4 n} \otimes F$ is a Clifford bundle, by the Atiyah-Singer index theorem $[5,20]$, the index of the twisted Dirac operators can be computed as

$$
\operatorname{ind}(\not \partial \otimes F)=\langle\widehat{A}(M) \operatorname{ch}(F),[M]\rangle,
$$

where $\operatorname{ch}(\cdot)$ denotes the Chern character, $\widehat{A}(M)$ denotes the $\widehat{A}$-genus, and $[M]$ denotes the fundamental cycle of $M$. In terms of formal roots,

$$
\begin{aligned}
& c(T M \otimes \mathbb{C})=\left(1+\eta_{1}\right)\left(1-\eta_{1}\right) \cdots\left(1+\eta_{2 n}\right)\left(1-\eta_{2 n}\right), \\
& p(T M)=\left(1+\eta_{1}^{2}\right) \cdots\left(1+\eta_{2 n}^{2}\right), \\
& c(F)=\left(1+\nu_{1}\right) \cdots\left(1+\nu_{p}\right), \\
& \operatorname{ch}(F)=\sum_{l=1} e^{\nu_{l}}, \\
& \operatorname{ind}(\not \partial \otimes F)=\left\langle\sum_{l=1} e^{\nu_{l}} \cdot \prod_{i=1}^{2 n} \frac{\eta_{i}}{e^{\frac{\eta_{i}}{2}}-e^{-\frac{\eta_{i}}{2}}},[M]\right\rangle .
\end{aligned}
$$

If $M$ admits a non-trivial $S^{1}$ action that lifts to $\Delta_{4 n} \otimes F$, the equivariant version of the index can be written in terms of the local data of the $S^{1}$-fixed point set $M^{S^{1}}$. More precisely, let $z \in S^{1}$ be a generic element of $S^{1}$. By the Atiyah-Singer fixed point theorem $[4,5]$

$$
\operatorname{ind}(\not \partial \otimes F)_{z}=\sum_{P \subset M^{S^{1}}} \mu(P, z)
$$

where $\mu(P, z)$ is the local contribution of the oriented fixed point submanifold $P \subset M^{S^{1}}$, which can be computed as follows. The $S^{1}$ action on $M$ induces a decomposition of $T M$ over $P$,

$$
\left.T M\right|_{P}=\sum_{k} N_{k},
$$

where $N_{k}$ is a bundle over $P$ whose fibers are representations of $S^{1}$ on which $z \in S^{1}$ acts as an automorphism with multiple eigenvalue $z^{k}, k \in \mathbb{Z}$. Note that $P$ inherits an orientation since $M$ is oriented and the bundles $N_{k}$ for $k \neq 0$ are naturally oriented. Formally, by means of the splitting principle, we can write

$$
\left.T M\right|_{P}=\mathcal{L}^{q_{1}}+\cdots+\mathcal{L}^{q_{2 n}},
$$

where $\mathcal{L}$ corresponds to the standard representation of $S^{1}$ on $\mathbb{C}$, so that $z \in S^{1}$ acts by multiplication by $z^{q_{i}}$ on $\mathcal{L}^{q_{i}}$. The integers $q_{i}=q_{i}(P) \in \mathbb{Z}$ are the exponents of the action at $P$, which correspond to the aforementioned numbers $k$. Thus, following [14, p. 67],

$$
\mu(P, z)=\left\langle\sum z^{-n_{k}} e^{\nu_{k}} \prod_{q_{i}=0} \frac{\eta_{i}}{e^{\frac{\eta_{i}}{2}}-e^{-\frac{\eta_{i}}{2}}} \prod_{q_{j} \neq 0} \frac{1}{z^{-\frac{q_{j}}{2}} e^{\frac{\eta_{j}}{2}}-z^{\frac{q_{j}}{2}} e^{-\frac{\eta_{j}}{2}}},[P]\right\rangle,
$$


where $n_{k}=n_{k}(P)$ are the exponents of the action on $F$ restricted to $P$. The function $\mu(P, z)$ is a rational function of the complex variable $z$ with zeroes at 0 and $\infty$ as long as

$$
\left|n_{k}\right|<\frac{1}{2}\left(\left|q_{1}(P)\right|+\cdots+\left|q_{2 n}(P)\right|\right)
$$

for all $1 \leq k \leq p$. If such a condition is fulfilled for all $P \subset M^{S^{1}}$, then $\operatorname{ind}(\not \partial \otimes F)_{z}$ is a rational function of $z$ with zeroes at 0 and $\infty$. Notice that $\operatorname{ind}(\not \partial \otimes F)_{z}$ also belongs to the representation $\operatorname{ring} R\left(S^{1}\right)$ of $S^{1}$, which can be identified with the Laurent polynomial ring $\mathbb{Z}\left[z, z^{-1}\right]$. Hence, by Lemma 2.5 ,

$$
\operatorname{ind}(\not \partial \otimes F)=\operatorname{ind}(\not \partial \otimes F)_{1}=0,
$$

i.e.,

$$
\langle\widehat{A}(M) \operatorname{ch}(F),[M]\rangle=0 .
$$

\section{4 $\widehat{A}$-genus of almost- $\mathrm{Cl}_{r}^{0}$-Hermitian manifolds}

Given a $4 n$-dimensional Riemannian manifold, according to the splitting principle with respect to the maxinal torus of $\mathrm{SO}(4 n)$, its complexified tangent bundle splits formally as follows

$$
T M \otimes \mathbb{C}=L_{1} \oplus L_{1}^{-1} \oplus \cdots \oplus L_{2 n} \oplus L_{2 n}^{-1}
$$

and, therefore,

$$
c(T M \otimes \mathbb{C})=\left(1+x_{1}\right)\left(1-x_{1}\right) \cdots\left(1+x_{2 n}\right)\left(1-x_{2 n}\right)=\left(1-x_{1}^{2}\right) \cdots\left(1-x_{2 n}^{2}\right)
$$

and its Pontrjagin class is

$$
p(T M)=\left(1+x_{1}^{2}\right) \cdots\left(1+x_{2 n}^{2}\right),
$$

and the $\widehat{A}$-genus is given by

$$
\widehat{A}(M)=\prod_{j=1}^{2 n} \frac{x_{i} / 2}{\sinh \left(x_{i} / 2\right)}=\prod_{j=1}^{2 n} \frac{x_{i}}{e^{x_{i} / 2}-e^{-x_{i} / 2}} .
$$

In the following, we will set $x_{i}=\eta_{i}$ from Section 3.1.

\subsection{1 $r \equiv 1,7(\bmod 8)$}

The $\widehat{A}$-genus is given by

$$
\widehat{A}(M)=\prod_{j=1}^{\left[\frac{m}{2}\right]} \prod_{k=1}^{\left[\frac{r}{2}\right]} \frac{\theta_{j}+\lambda_{k}}{e^{\frac{\theta_{j}+\lambda_{k}}{2}}-e^{-\frac{\theta_{j}+\lambda_{k}}{2}}}
$$

if $m$ is even, and

$$
\widehat{A}(M)=\prod_{j=1}^{\left[\frac{m}{2}\right]} \prod_{k=1}^{2^{\left[\frac{r}{2}\right]}} \frac{\theta_{j}+\lambda_{k}}{e^{\frac{\theta_{j}+\lambda_{k}}{2}}-e^{-\frac{\theta_{j}+\lambda_{k}}{2}}} \prod_{l=1}^{2^{\left[\frac{r}{2}\right]-1}} \frac{\lambda_{l}}{e^{\frac{\lambda_{l}}{2}}-e^{-\frac{\lambda_{l}}{2}}}
$$

if $m$ is odd. 


\subsection{2 $r \equiv 2,6(\bmod 8)$}

The $\widehat{A}$-genus is given by

$$
\widehat{A}(M)= \begin{cases}\prod_{j=1}^{m} \prod_{k=1}^{2^{\frac{r}{2}-1}} \frac{\theta_{j}+\lambda_{k}^{+}}{e^{\frac{\theta_{j}+\lambda_{k}^{+}}{2}}-e^{-\frac{\theta_{j}+\lambda_{k}^{+}}{2}}} & \text { if } r \equiv 2(\bmod 8), \\ \prod_{j=1}^{m} \prod_{k=1}^{2^{\frac{r}{2}-1}} \frac{\theta_{j}+\lambda_{k}^{-}}{e^{\frac{\theta_{j}+\lambda_{k}^{-}}{2}}-e^{-\frac{\theta_{j}+\lambda_{k}^{-}}{2}}} & \text { if } r \equiv 6(\bmod 8) .\end{cases}
$$

\subsection{3 $r \equiv 3,5(\bmod 8)$}

The $\widehat{A}$-genus is given by

$$
\widehat{A}(M)=\prod_{j=}^{m} \prod_{k=1}^{2^{\left[\frac{r}{2}\right]}} \frac{\theta_{j}+\lambda_{k}}{e^{\frac{\theta_{j}+\lambda_{k}}{2}}-e^{-\frac{\theta_{j}+\lambda_{k}}{2}}} .
$$

\subsection{4 $r \equiv 4(\bmod 8)$}

The $\widehat{A}$-genus is given by

$$
\widehat{A}(M)=\prod_{j_{1}=1}^{m_{1}} \prod_{k=1}^{2^{\frac{r}{2}-1}} \frac{\theta_{j_{1}}+\lambda_{k}^{+}}{e^{\frac{\theta_{j_{1}}+\lambda_{k}^{+}}{2}}-e^{-\frac{\theta_{j_{1}}+\lambda_{k}^{+}}{2}}} \prod_{j_{2}=1}^{m_{2}} \prod_{k=1}^{2^{\frac{r}{2}-1}} \frac{\theta_{j_{2}}^{\prime}+\lambda_{k}^{-}}{e^{\frac{\theta_{j_{2}}^{\prime}+\lambda_{k}^{-}}{2}}-e^{-\frac{\theta_{j_{2}}^{\prime}+\lambda_{k}^{-}}{2}}} .
$$

\subsection{5 $r \equiv 0(\bmod 8)$}

We can set

- if $m_{1}, m_{2}$ are even,

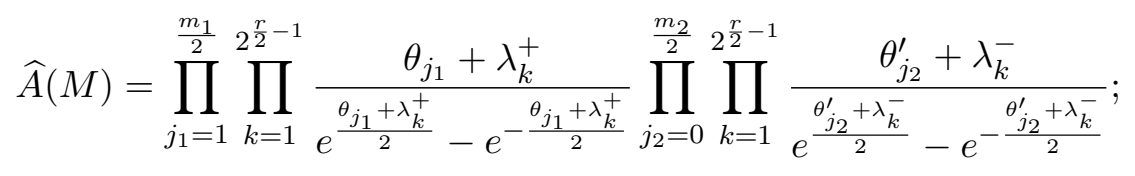

- if $m_{1}$ is even and $m_{2}$ is odd,

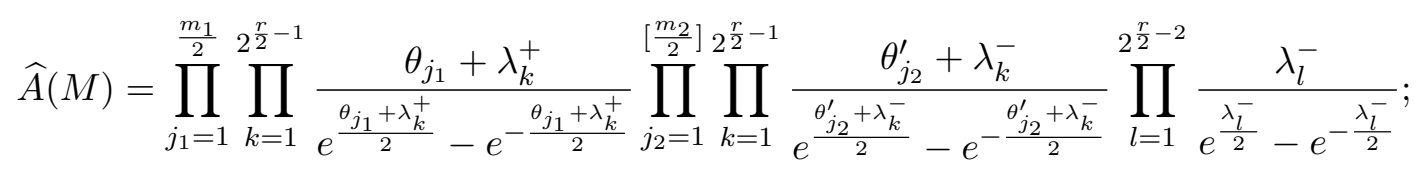

- if $m_{1}$ is odd and $m_{2}$ is even,

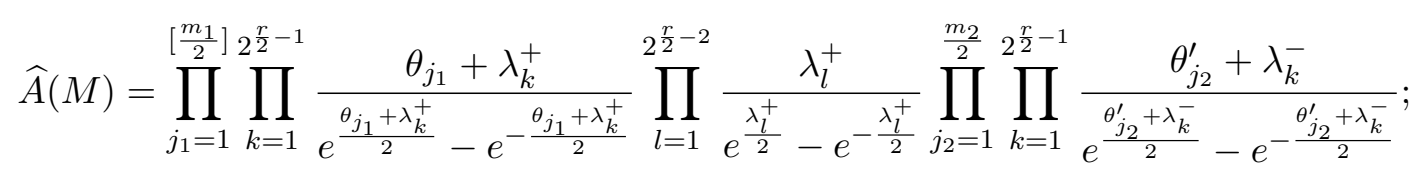

- if $m_{1}, m_{2}$ are odd,

$$
\begin{aligned}
& \widehat{A}(M)=\prod_{j_{1}=1}^{\left[\frac{m_{1}}{2}\right]} \prod_{k=1}^{2^{\frac{r}{2}-1}} \frac{\theta_{j_{1}}+\lambda_{k}^{+}}{e^{\frac{\theta_{j_{1}}+\lambda_{k}^{+}}{2}}-e^{-\frac{\theta_{j_{1}}+\lambda_{k}^{+}}{2}}} \prod_{l=1}^{2^{\frac{r}{2}-2}} \frac{\lambda_{l}^{+}}{e^{\frac{\lambda_{l}^{+}}{2}}-e^{-\frac{\lambda_{l}^{+}}{2}}}
\end{aligned}
$$

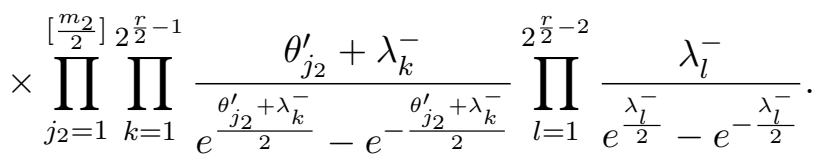




\subsection{Infinitesimal automorphisms}

An automorphism of an almost- $\mathrm{Cl}_{r}^{0}$-Hermitian manifold $M$ is an isometry which preserves the almost even-Clifford Hermitian structure. A vector field $X$ on $M$ is an infinitesimal automorphism if it is a Killing vector field that preserves the structure, i.e., locally

$$
\mathcal{L}_{X} J_{i j}=\sum_{k<l} \alpha_{k l}^{(i j)} J_{k l}
$$

for some (local) functions $\alpha_{k l}^{(i j)}$, where $\mathcal{L}_{X}$ denotes the Lie derivative in the direction of $X$. Consider

$$
\mathcal{L}_{X}\left(J_{i j}(Y)\right)=\left(\mathcal{L}_{X} J_{i j}\right)(Y)+J_{i j}\left(\mathcal{L}_{X} Y\right),
$$

which can be written in terms of the Levi-Civita connection $\nabla$ as follows

$$
\nabla_{X}\left(J_{i j}(Y)\right)-\nabla_{J_{i j}(Y)} X=\sum_{k<l} \alpha_{k l}^{(i j)} J_{k l}(Y)+J_{i j}\left(\nabla_{X} Y-\nabla_{Y} X\right)
$$

Now, if $p \in M$ is such that $X_{p}=0$,

$$
-\nabla_{J_{i j}(Y)} X=\sum_{k<l} \alpha_{k l}^{(i j)} J_{k l}(Y)-J_{i j}\left(\nabla_{Y} X\right)
$$

i.e.,

$$
\left[J_{i j}, \nabla X\right](Y)=\sum_{k<l} \alpha_{k l}^{(i j)} J_{k l}(Y)
$$

Hence, $(\nabla X)_{p}$ is a skew-symmetric endomorphism such that

$$
\left[J_{i j}, \nabla X\right]=\sum_{k<l} \alpha_{k l}^{(i j)} J_{k l} .
$$

i.e., $(\nabla X)_{p}$ belongs to Lie algebra of the structure group of $M[1,2]$.

We will say that a smooth circle action on an almost- $\mathrm{Cl}_{r}^{0}$-Hermitian manifold is an action by automorphisms if the corresponding Killing vector field is an infinitesimal automorphism.

Example 4.6. The 16-dimensional symmetric space

$$
\frac{F_{4}}{\operatorname{Spin}(9)}
$$

has an almost- $\mathrm{Cl}_{9}^{0}$-Hermitian structure admitting $S^{1}$ actions by automorphisms [10].

\subsection{Exponents of the $S^{1}$ action}

In this section, let $M$ be a compact, rank $r$ almost even-Clifford Hermitian manifold with a nontrivial (effective) $S^{1}$ action by automorphisms. Let $P \subset M^{S^{1}}$ be an $S^{1}$-fixed submanifold. The corresponding infinitesimal isometry $X$ is such that $(\nabla X)_{p} \in \mathfrak{s o}(N)$ at any fixed point $p \in P$. This corresponds to the induced action of $S^{1}$ to $T_{p} M$, and such a circle lies in a maximal torus. The tangent space at $p$ decomposes as in Section 4.3. In fact, we can now be more precise about these exponents. By Section $4.5,(\nabla X)_{p}$ belongs to a Cartan subalgebra of the Lie algebra of the structure group, and we can assume that the decomposition (4.1) is compatible with a decomposition such as (4.2) into complex lines with respect to a maximal torus of such 
a group. Hence, we can read off the exponents of the action with respect to the weights given in Section 3.1:

\begin{tabular}{|c|c|c|c|}
\hline$r(\bmod 8)$ & $\pm q_{i}$ & & \\
\hline 0 & $\frac{t_{j_{1}} \pm h_{k}^{+}}{2}, \frac{t_{j_{2}}^{\prime} \pm h_{k}^{-}}{2}$ & $1 \leq j_{1} \leq\left[\frac{m_{1}}{2}\right]$, & $1 \leq k \leq 2^{\left[\frac{r}{2}\right]-2}$ \\
& $\left(\frac{h_{l}}{2}, \frac{h^{\frac{r}{2}-1}+l}{2}\right)$ & $1 \leq j_{2} \leq\left[\frac{m_{2}}{2}\right]$ & $\left(1 \leq l \leq 2^{\left[\frac{r}{2}\right]-3}\right)$ \\
\hline 1,7 & $\frac{t_{j} \pm h_{k}}{2}\left(\frac{h_{l}}{2}\right)$ & $1 \leq j \leq\left[\frac{m}{2}\right]$ & $\begin{array}{c}1 \leq k \leq 2^{\left[\frac{r}{2}\right]-1} \\
\left(\leq l \leq 2^{\left[\frac{r}{2}\right]-2}\right)\end{array}$ \\
\hline 2,6 & $\frac{t_{j} \pm h_{k}^{+}}{2}, \frac{-t_{j} \pm h_{k}^{-}}{2}$ & $1 \leq j \leq m$ & $1 \leq k \leq 2^{\left[\frac{r}{2}\right]-2}$ \\
\hline 3,5 & $\frac{t_{j} \pm h_{k}}{2}$ & $1 \leq j \leq m$ & $1 \leq k \leq 2^{\left[\frac{r}{2}\right]-1}$ \\
\hline 4 & $\frac{t_{j_{1} \pm h_{k}^{+}}}{2}, \frac{t_{j_{2}}^{\prime} \pm h_{k}^{-}}{2}$ & $1 \leq j_{1} \leq m_{1}, 1 \leq j_{2} \leq m_{2}$ & $1 \leq k \leq 2^{\left[\frac{r}{2}\right]-2}$ \\
\hline
\end{tabular}

where $m, m_{1}, m_{2}$ denote the corresponding multiplicities. Here, the numbers $\frac{t_{j}}{2}, \frac{t_{j}^{\prime}}{2}$ are the exponents corresponding to the complex representations $E, E_{1}, E_{2}$ described in Section 2.3.1, $f_{j}$ are the exponents for the $\mathrm{SO}(r)$ representation for $r$ odd or $\mathbb{P S O}(r)$ representation for $r$ even, $h_{k}$ denote the numbers

$$
\pm f_{1} \pm \cdots \pm f_{\left[\frac{r}{2}\right]}
$$

in some order for $r$ odd, and $h_{k}^{ \pm}$denote the numbers

$$
\pm f_{1} \pm \cdots \pm f_{\frac{r}{2}}
$$

with an even or odd number of negative signs respectively, listed in some order for $r$ even.

\subsection{Vanishing theorems}

In this section, we give the main details of the proofs of the vanishing theorems.

Theorem 4.7. Let $M$ be a compact $N$-dimensional almost- $\mathrm{Cl}_{r}^{0}$-Hermitian admitting a smooth circle action by automorphisms, $r \geq 3$. Let $E, E_{1}, E_{2}$ be the (locally defined) bundles described in (2.4), $m, m_{1}, m_{2}$ the corresponding multiplicities and $u, u_{1}, u_{2}, s, t$ be non-negative integers satisfying the conditions given in Sections 3.2 and 3.3. Then,

- for $r \equiv 1,7(\bmod 8)$, if $0 \leq u+s<\left[\frac{m}{2}\right]$,

$$
\left\langle\operatorname{ch}\left(\bigwedge^{u} E\right) \operatorname{ch}\left(\Delta_{r}\right)^{s} \widehat{A}(M),[M]\right\rangle=0 ;
$$

- for $r \equiv 3,5(\bmod 8)$, if $0 \leq u+s<m$,

$$
\left\langle\operatorname{ch}\left(\bigwedge^{u} E\right) \operatorname{ch}\left(\Delta_{r}\right)^{s} \widehat{A}(M),[M]\right\rangle=0 ;
$$

- for $r \equiv 0(\bmod 8)$, if $0 \leq u_{1}+s<\left[\frac{m_{1}}{2}\right], 0 \leq u_{2}+t<\left[\frac{m_{2}}{2}\right]$,

$$
\left\langle\operatorname{ch}\left(\bigwedge^{u_{1}} E_{1}\right) \operatorname{ch}\left(\bigwedge^{u_{2}} E_{2}\right) \operatorname{ch}\left(\Delta_{r}^{+}\right)^{s} \operatorname{ch}\left(\Delta_{r}^{-}\right)^{t} \widehat{A}(M),[M]\right\rangle=0
$$

- for $r \equiv 2,6(\bmod 8)$, if $0 \leq u_{1}+s<m$ and $0 \leq u_{2}+t<m$, or if $0 \leq u_{1}+t<m$ and $0 \leq u_{2}+s<m$

$$
\left\langle\operatorname{ch}\left(\bigwedge^{u_{1}} E\right) \operatorname{ch}\left(\bigwedge^{u_{2}} \bar{E}\right) \operatorname{ch}\left(\Delta_{r}^{+}\right)^{s} \operatorname{ch}\left(\Delta_{r}^{-}\right)^{t} \widehat{A}(M),[M]\right\rangle=0
$$


- for $r \equiv 4(\bmod 8)$, if $0 \leq u_{1}+s<m_{1}$ and $0 \leq u_{2}+t<m_{2}$,

$$
\left\langle\operatorname{ch}\left(\bigwedge^{u_{1}} E_{1}\right) \operatorname{ch}\left(\bigwedge^{u_{2}} E_{2}\right) \operatorname{ch}\left(\Delta_{r}^{+}\right)^{s} \operatorname{ch}\left(\Delta_{r}^{-}\right)^{t} \widehat{A}(M),[M]\right\rangle=0 .
$$

If the inequalities are not strict, the indices are rigid.

Proof. Since the $S^{1}$ action is by automorphisms of the almost even-Clifford Hermitian structure, the action lifts to the bundles associated to the structure group, such as the twisted spin bundles we are considering. Given that the arguments are similar in all cases, we will only describe the calculation for $r \equiv 1,7(\bmod 8)$ and $m$ even.

Let $P \subset M^{S^{1}}$ be an $S^{1}$-fixed submanifold. By Section 4.5 , over $P$ the circle group of automorphisms maps non-trivially to the structure group $\mathrm{SO}(m) \operatorname{Spin}(r)$, so that the fibers of the bundles $\Delta_{N} \otimes \bigwedge^{u} E \otimes \Delta_{r}^{\otimes s}$ over points of $P$ decompose as sums of representations of $S^{1}$. Recall that

$$
\operatorname{ch}\left(\bigwedge^{u} E\right)=\sum_{1 \leq i_{1}<\cdots<i_{u} \leq 2\left[\frac{m}{2}\right]} e^{\vartheta_{i_{1}}+\cdots+\vartheta_{i_{u}}}
$$

where

$$
\vartheta_{j}=\theta_{j}, \quad \vartheta_{[m / 2]+j}=-\theta_{j}, \quad j=1, \ldots,[m / 2] .
$$

Thus, the exponents of the twist will be of the form

$$
\frac{1}{2}\left(\sum_{a=1}^{c}(-1)^{\varepsilon_{a}} t_{i_{a}}+\sum_{b=1}^{s}(-1)^{\delta_{b}} h_{l_{b}}\right),
$$

where $0 \leq c \leq u, \varepsilon_{a}, \delta_{b} \in\{0,1\}$. There are two points to verify in the proof: firstly, that the contributions $\mu(P, z)$ are rational functions and, secondly, that the exponents of the twisting bundles and the tangent space satisfy the inequality (4.3).

The first one follows from the fact that the fibers of the bundles $\Delta_{N} \otimes \bigwedge^{u} E \otimes \Delta_{r}^{\otimes s}$ over $P$ decompose as sums of representations of $S^{1}$. Formally, according to the splitting principle, if

$$
T M_{c}=L_{1} \oplus L_{1}^{-1} \oplus \cdots \oplus L_{N / 2} \oplus L_{N / 2}^{-1}
$$

then

$$
\begin{aligned}
\Delta_{N} & =\left(L_{1}^{1 / 2} \oplus L_{1}^{-1 / 2}\right) \otimes \cdots \otimes\left(L_{N / 2}^{1 / 2} \oplus L_{N / 2}^{-1 / 2}\right) \\
& =L_{1}^{1 / 2} \otimes \cdots \otimes L_{N / 2}^{1 / 2} \oplus \cdots \oplus L_{1}^{-1 / 2} \otimes \cdots \otimes L_{N / 2}^{-1 / 2}
\end{aligned}
$$

so that the $S^{1}$-exponents on these lines will be of the form

$$
\sum\left( \pm \frac{t_{j} \pm h_{k}}{4}\right)+\sum\left( \pm \frac{h_{l}}{4}\right)
$$

The bundle $\Delta_{N} \otimes \bigwedge^{u} E \otimes \Delta_{r}^{\otimes s}$ will have integer exponents over $P$ of the form

$$
\begin{gathered}
\frac{1}{2}\left(\sum_{a=1}^{c}(-1)^{\varepsilon_{a}} t_{i_{a}}+\sum_{b=1}^{s}(-1)^{\delta_{b}} h_{l_{b}}\right)+\sum_{q_{j} \neq 0}\left((-1)^{\gamma_{j}} \frac{t_{j}+h_{k}}{4}\right) \\
+\sum_{q_{j^{\prime}} \neq 0}\left((-1)^{\gamma_{j^{\prime}}} \frac{t_{t_{j}}-h_{k}}{4}\right)+\sum_{q_{l} \neq 0}\left((-1)^{\zeta_{l}} \frac{h_{l}}{4}\right) .
\end{gathered}
$$


Thus, the powers of $z$ in each summand of $\mu(P, z)$ can be rearranged in order to show that such a summand is a product of rational functions such as the one described in Lemma 2.5.

For the second point, it is sufficient to consider the exponents of the form

$$
\frac{1}{2}\left(\sum_{a=1}^{u}(-1)^{\varepsilon_{a}} t_{i_{a}} \pm s h_{k}\right) .
$$

Since $u+s<\left[\frac{m}{2}\right]$, there exists an $s$-tuple of indices $j_{1}<\cdots<j_{s}$ such that $\left\{j_{1}, \ldots, j_{s}\right\} \subset$ $\left\{1, \ldots,\left[\frac{m}{2}\right]\right\}-\left\{i_{1}, \ldots, i_{u}\right\}$. Thus,

$$
\begin{aligned}
\left|\sum_{a=1}^{u}(-1)^{\varepsilon_{a}} t_{i_{a}} \pm s h_{k}\right| & =\left|\sum_{a=1}^{u}(-1)^{\varepsilon_{a}}\left(\frac{t_{i_{a}}+h_{k}}{2}+\frac{t_{i_{a}}-h_{k}}{2}\right) \pm \sum_{b=1}^{s}\left(\frac{h_{k}+t_{j_{b}}}{2}+\frac{h_{k}-t_{j_{b}}}{2}\right)\right| \\
& \leq \sum_{a=1}^{u}\left(\left|\frac{t_{i_{a}}+h_{k}}{2}\right|+\left|\frac{t_{i_{a}}-h_{k}}{2}\right|\right)+\sum_{b=1}^{s}\left(\left|\frac{h_{k}+t_{j_{b}}}{2}\right|+\left|\frac{h_{k}-t_{j_{b}}}{2}\right|\right) \\
& \leq \sum_{i=1}^{m}\left|\frac{t_{i}+h_{k}}{2}\right|+\left|\frac{t_{i}-h_{k}}{2}\right|<\sum_{l=1}^{2^{[r / 2]-1}} \sum_{i=1}^{m}\left|\frac{t_{i}+h_{l}}{2}\right|+\left|\frac{t_{i}-h_{l}}{2}\right| \\
& \leq \sum_{c=1}^{N / 2}\left|q_{c}\right|,
\end{aligned}
$$

which is the corresponding version of the inequality (4.3) in Section 4.3

Theorem 4.8. Let $M$ be a compact $N$-dimensional almost- $\mathrm{Cl}_{r}^{0}$-Hermitian admitting a smooth circle action by automorphisms, $r \geq 3$. Let $E, E_{1}, E_{2}$ be the (locally defined) bundles described in (2.4), $m, m_{1}, m_{2}$ the corresponding multiplicities and $u, u_{1}, u_{2}, s, t$ be non-negative integers satisfying the conditions given in Sections 3.2 and 3.3. Then,

- for $r \equiv 1,7(\bmod 8)$, if $0 \leq u+s<\left[\frac{m}{2}\right]$ and $u \leq 2^{\left[\frac{r}{2}\right]-1}$,

$$
\left\langle\operatorname{ch}\left(S^{u} E\right) \operatorname{ch}\left(\Delta_{r}\right)^{s} \widehat{A}(M),[M]\right\rangle=0
$$

- for $r \equiv 3,5(\bmod 8)$, if $0 \leq u+s<m$ and $u \leq 2^{\left[\frac{r}{2}\right]-1}$,

$$
\left\langle\operatorname{ch}\left(S^{u} E\right) \operatorname{ch}\left(\Delta_{r}\right)^{s} \widehat{A}(M),[M]\right\rangle=0 ;
$$

- for $r \equiv 0(\bmod 8)$, if $0 \leq u_{1}+s<\left[\frac{m_{1}}{2}\right], 0 \leq u_{2}+t<\left[\frac{m_{2}}{2}\right]$ and $u_{1}, u_{2} \leq 2^{\left[\frac{r}{2}\right]-2}$,

$$
\left\langle\operatorname{ch}\left(S^{u_{1}} E_{1}\right) \operatorname{ch}\left(S^{u_{2}} E_{2}\right) \operatorname{ch}\left(\Delta_{r}^{+}\right)^{s} \operatorname{ch}\left(\Delta_{r}^{-}\right)^{t} \widehat{A}(M),[M]\right\rangle=0
$$

- for $r \equiv 2,6(\bmod 8)$, if $u_{1}, u_{2} \leq 2^{\left[\frac{r}{2}\right]-2}$ and one of $0 \leq u_{1}+s<m, 0 \leq u_{2}+t<m$ or $0 \leq u_{1}+t<m, 0 \leq u_{2}+s<m$,

$$
\left\langle\operatorname{ch}\left(S^{u_{1}} E\right) \operatorname{ch}\left(S^{u_{2}} \bar{E}\right) \operatorname{ch}\left(\Delta_{r}^{+}\right)^{s} \operatorname{ch}\left(\Delta_{r}^{-}\right)^{t} \widehat{A}(M),[M]\right\rangle=0 ;
$$

- for $r \equiv 4(\bmod 8)$, if $0 \leq u_{1}+s<m_{1}, 0 \leq u_{2}+t<m_{2}$ and $u_{1}, u_{2} \leq 2^{\left[\frac{r}{2}\right]-2}$,

$$
\left\langle\operatorname{ch}\left(S^{u_{1}} E_{1}\right) \operatorname{ch}\left(S^{u_{2}} E_{2}\right) \operatorname{ch}\left(\Delta_{r}^{+}\right)^{s} \operatorname{ch}\left(\Delta_{r}^{-}\right)^{t} \widehat{A}(M),[M]\right\rangle=0 .
$$

If the inequalities are not strict, the indices are rigid. 
Proof. We will only describe the relevant changes to the calculation for $r \equiv 1,7(\bmod 8)$ and $m$ even. Let $P \subset M^{S^{1}}$ be an $S^{1}$-fixed submanifold. Recall that

$$
\operatorname{ch}\left(S^{u} E\right)=\sum_{1 \leq i_{1} \leq \cdots \leq i_{u} \leq 2\left[\frac{m}{2}\right]} e^{\vartheta_{i_{1}}+\cdots+\vartheta_{i_{u}}},
$$

where

$$
\vartheta_{j}=\theta_{j}, \quad \vartheta_{[m / 2]+j}=-\theta_{j}, \quad j=1, \ldots,[m / 2] .
$$

Thus, the exponents of the twist will be of the form

$$
\frac{1}{2}\left(\sum_{a=1}^{u}(-1)^{\varepsilon_{a}} t_{i_{a}}+\sum_{b=1}^{s}(-1)^{\delta_{b}} h_{l_{b}}\right),
$$

where $\varepsilon_{a}, \delta_{b} \in\{0,1\}$. It is sufficient to consider the exponents of the form

$$
\frac{1}{2}\left(\sum_{a=1}^{u}(-1)^{\varepsilon_{a}} t_{i_{a}} \pm s h_{k}\right) .
$$

Among them, there are two extreme types, namely the ones equal to exponents of the exterior powers which we already know how to deal with, and the ones such as $u t_{1}$. For such an exponent, consider

$$
\begin{aligned}
\left|u t_{1}\right| & =\left|\sum_{l=1}^{u} \frac{t_{1}+h_{l}}{2}+\frac{t_{1}-h_{l}}{2}\right| \leq \sum_{l=1}^{u}\left|\frac{t_{1}+h_{l}}{2}\right|+\left|\frac{t_{1}-h_{l}}{2}\right| \leq \sum_{l=1}^{2^{\left[\frac{r}{2}\right]-1}}\left|\frac{t_{1}+h_{l}}{2}\right|+\left|\frac{t_{1}-h_{l}}{2}\right| \\
& <\sum_{j=1}^{\left[\frac{m}{2}\right]} \sum_{l=1}^{\left[{ }^{\left[\frac{r}{2}\right]-1}\right.}\left|\frac{t_{1}+h_{l}}{2}\right|+\left|\frac{t_{1}-h_{l}}{2}\right| \leq \sum_{c=1}^{N / 2}\left|q_{c}\right|,
\end{aligned}
$$

if $u<2^{[r / 2]-1}$.

Theorem 4.9. Let $M$ be a compact $N$-dimensional almost- $\mathrm{Cl}_{r}^{0}$-Hermitian admitting a smooth circle action by automorphisms, $r \geq 3$. Let $E, E_{1}, E_{2}$ be the (locally defined) bundles described in (2.4), $m, m_{1}, m_{2}$ the corresponding multiplicities and $u_{i}, v_{i}, u_{i}^{\prime}, v_{i}^{\prime}, s, t$ be non-negative integers satisfying analogous conditions to those given in Sections 3.2 and 3.3. Then,

- for $r \equiv 1,7(\bmod 8)$, if

$$
0 \leq \sum_{i=1}^{b} u_{i}+\sum_{j=1}^{b} v_{j}+s<\left[\frac{m}{2}\right]
$$

and

$$
\begin{aligned}
& a+\sum_{i=1}^{b} v_{i} \leq 2^{\left[\frac{r}{2}\right]-1} \\
& \left\langle\operatorname{ch}\left(\bigotimes_{i=1}^{a} \bigwedge^{u_{i}} E \otimes \bigotimes_{j=1}^{b} S^{v_{j}} E \otimes\left(\Delta_{r}\right)^{\otimes s}\right) \widehat{A}(M),[M]\right\rangle=0
\end{aligned}
$$


- for $r \equiv 3,5(\bmod 8)$, if

$$
0 \leq \sum_{i=1}^{b} u_{i}+\sum_{j=1}^{b} v_{j}+s<m
$$

and

$$
\begin{aligned}
& a+\sum_{i=1}^{b} v_{i} \leq 2^{\left[\frac{r}{2}\right]-1} \\
& \left\langle\operatorname{ch}\left(\bigotimes_{i=1}^{a} \bigwedge^{u_{i}} E \otimes \bigotimes_{j=1}^{b} S^{v_{j}} E \otimes\left(\Delta_{r}\right)^{\otimes s}\right) \widehat{A}(M),[M]\right\rangle=0
\end{aligned}
$$

- for $r \equiv 0(\bmod 8)$, if

$$
0 \leq \sum_{i=1}^{b} u_{i}+\sum_{j=1}^{b} v_{j}+s<\left[\frac{m_{1}}{2}\right], \quad 0 \leq \sum_{i=1}^{c} u_{i}^{\prime}+\sum_{j=1}^{d} v_{j}^{\prime}+t<\left[\frac{m_{2}}{2}\right]
$$

and

$$
\begin{aligned}
& a+\sum_{i=1}^{b} v_{i}, c+\sum_{i=1}^{d} v_{i}^{\prime} \leq 2^{\left[\frac{r}{2}\right]-2} \\
& \left\langle\operatorname { c h } \left(\bigotimes_{i=1}^{a} \wedge^{u_{i}} E_{1} \otimes \bigotimes_{j=1}^{b} S^{v_{j}} E_{1} \otimes \bigotimes_{k=1}^{c} \wedge^{u_{k}^{\prime}} E_{2} \otimes \bigotimes_{l=1}^{d} S^{v_{l}^{\prime}} E_{2}\right.\right. \\
& \left.\left.\otimes\left(\Delta_{r}^{+}\right)^{\otimes s} \otimes\left(\Delta_{r}^{-}\right)^{\otimes t}\right) \widehat{A}(M),[M]\right\rangle=0
\end{aligned}
$$

- for $r \equiv 2(\bmod 8)$, if

$$
a+\sum_{i=1}^{b} v_{i}, \quad c+\sum_{i=1}^{d} v_{i}^{\prime} \leq 2^{\left[\frac{r}{2}\right]-2}
$$

and

$$
0 \leq \sum_{i=1}^{b} u_{i}+\sum_{j=1}^{b} v_{j}+s<m, \quad 0 \leq \sum_{i=1}^{c} u_{i}^{\prime}+\sum_{j=1}^{d} v_{j}^{\prime}+t<m
$$

or

$$
\begin{gathered}
0 \leq \sum_{i=1}^{b} u_{i}+\sum_{j=1}^{b} v_{j}+t<m, \quad 0 \leq \sum_{i=1}^{c} u_{i}^{\prime}+\sum_{j=1}^{d} v_{j}^{\prime}+s<m \\
\left\langle\operatorname { c h } \left(\bigotimes_{i=1}^{a} \bigwedge^{u_{i}} E \otimes \bigotimes_{j=1}^{b} S^{v_{j}} E \otimes \bigotimes_{k=1}^{c} \bigwedge^{u_{k}^{\prime}} \bar{E} \otimes \bigotimes_{l=1}^{d} S^{v_{l}^{\prime}} \bar{E}\right.\right. \\
\left.\left.\otimes\left(\Delta_{r}^{+}\right)^{\otimes s} \otimes\left(\Delta_{r}^{-}\right)^{\otimes t}\right) \widehat{A}(M),[M]\right\rangle=0
\end{gathered}
$$


- for $r \equiv 4(\bmod 8)$, if

$$
0 \leq \sum_{i=1}^{b} u_{i}+\sum_{j=1}^{b} v_{j}+s<m_{1}, \quad 0 \leq \sum_{i=1}^{c} u_{i}^{\prime}+\sum_{j=1}^{d} v_{j}^{\prime}+t<m_{2}
$$

and

$$
\begin{aligned}
& a+\sum_{i=1}^{b} v_{i}, \quad c+\sum_{i=1}^{d} v_{i}^{\prime} \leq 2^{\left[\frac{r}{2}\right]-2} \\
& \left\langle\operatorname { c h } \left(\bigotimes_{i=1}^{a} \wedge^{u_{i}} E_{1} \otimes \bigotimes_{j=1}^{b} S^{v_{j}} E_{1} \otimes \bigotimes_{k=1}^{c} \wedge^{u_{k}^{\prime}} E_{2} \otimes \bigotimes_{l=1}^{d} S^{v_{l}^{\prime}} E_{2}\right.\right. \\
& \left.\left.\otimes\left(\Delta_{r}^{+}\right)^{\otimes s} \otimes\left(\Delta_{r}^{-}\right)^{\otimes t}\right) \widehat{A}(M),[M]\right\rangle=0 .
\end{aligned}
$$

If the inequalities are not strict, the indices are rigid.

Remark 4.10. When $r=3$, Theorems 4.7 and 4.8 return the vanishings for almost quaternionHermitian manifolds proved in [12].

Remark 4.11. Theorems 4.7, 4.8 and 4.9 do not restrict to the well known vanishings for almost Hermitian manifolds proved in [11], which require a divisibility condition on $c_{1}(M)$. This is due to the fact that the structure group of a $2 m$-dimensional almost Hermitian manifold is $\mathrm{U}(m)$ instead of

$$
\frac{\mathrm{U}(m) \times \operatorname{Spin}(2)}{\left\{ \pm\left(\operatorname{Id}_{2}, 1\right), \pm\left(i \mathrm{Id}_{2},-\mathrm{vol}_{2}\right)\right\}}
$$

Remark 4.12. For $r \neq 3,4,6,8$, an almost- $\mathrm{Cl}_{r}^{0}$-Hermitian manifold is Spin (see [1, Theorem 4.1]). Thus, for $u=u_{1}=u_{2}=s=t=0$, the vanishings in the theorems restrict to Atiyah-Hirzebruch's vanishing.

\section{Acknowledgements}

The first named author was supported by CONACyT. The second named author was partially supported by a CONACyT grant. The second named author wishes to thank the International Centre for Theoretical Physics and the Institut des Hautes Études Scientifiques for their hospitality and support. We would like to express our gratitude to the anonymous referees for their careful reading of this manuscript and their helpful comments.

\section{References}

[1] Arizmendi G., Garcia-Pulido A.L., Herrera R., A note on the geometry and topology of almost even-Clifford Hermitian manifolds, arXiv:1606.00774.

[2] Arizmendi G., Herrera R., Centralizers of spin subalgebras, J. Geom. Phys. 97 (2015), 77-92, arXiv:1503.06168.

[3] Atiyah M.F., Hirzebruch F., Spin-manifolds and group actions, in Essays on Topology and Related Topics (Mémoires dédiés à Georges de Rham), Springer, New York, 1970, 18-28.

[4] Atiyah M.F., Singer I.M., The index of elliptic operators. III, Ann. of Math. 87 (1968), 546-604.

[5] Berline N., Getzler E., Vergne M., Heat kernels and Dirac operators, Grundlehren Text Editions, SpringerVerlag, Berlin, 2004.

[6] Bott R., Taubes C., On the rigidity theorems of Witten, J. Amer. Math. Soc. 2 (1989), 137-186. 
[7] Bröcker T., tom Dieck T., Representations of compact Lie groups, Graduate Texts in Mathematics, Vol. 98, Springer-Verlag, New York, 1995.

[8] Dessai A., Rigidity theorems for Spin ${ }^{\mathbf{C}}$-manifolds, Topology 39 (2000), 239-258.

[9] Friedrich T., Dirac operators in Riemannian geometry, Graduate Studies in Mathematics, Vol. 25, Amer. Math. Soc., Providence, RI, 2000.

[10] Friedrich T., Weak Spin(9)-structures on 16-dimensional Riemannian manifolds, Asian J. Math. 5 (2001), 129-160, math.DG/9912112.

[11] Hattori A., Spin ${ }^{c}$-structures and $S^{1}$-actions, Invent. Math. 48 (1978), 7-31.

[12] Herrera H., Herrera R., Rigidity and vanishing theorems for almost quaternionic manifolds, Geom. Dedicata 134 (2008), 139-152.

[13] Hirzebruch F., Elliptic genera of level $N$ for complex manifolds, in Differential Geometrical Methods in Theoretical Physics (Como, 1987), NATO Adv. Sci. Inst. Ser. C Math. Phys. Sci., Vol. 250, Kluwer Acad. Publ., Dordrecht, 1988, 37-63.

[14] Hirzebruch F., Berger T., Jung R., Manifolds and modular forms, Aspects of Mathematics, Vol. E20, Friedr. Vieweg \& Sohn, Braunschweig, 1992.

[15] Hirzebruch F., Slodowy P., Elliptic genera, involutions, and homogeneous spin manifolds, Geom. Dedicata 35 (1990), 309-343.

[16] LeBrun C., Salamon S., Strong rigidity of positive quaternion-Kähler manifolds, Invent. Math. 118 (1994), 109-132.

[17] Lichnerowicz A., Spineurs harmoniques, C. R. Acad. Sci. Paris 257 (1963), 7-9.

[18] Liu K., On modular invariance and rigidity theorems, J. Differential Geom. 41 (1995), 343-396.

[19] Moroianu A., Semmelmann U., Clifford structure on Riemannian manifolds, Adv. Math. 228 (2011), 940967, arXiv:0912.4207.

[20] Roe J., Elliptic operators, topology and asymptotic methods, Pitman Research Notes in Mathematics Series, Vol. 395, 2nd ed., Longman, Harlow, 1998.

[21] Salamon S., Riemannian geometry and holonomy groups, Pitman Research Notes in Mathematics Series, Vol. 201, Longman, Harlow, 1989.

[22] Taubes C.H., $S^{1}$ actions and elliptic genera, Comm. Math. Phys. 122 (1989), 455-526.

[23] Witten E., Elliptic genera and quantum field theory, Comm. Math. Phys. 109 (1987), 525-536.

[24] Witten E., The index of the Dirac operator in loop space, in Elliptic Curves and Modular Forms in Algebraic Topology (Princeton, NJ, 1986), Lecture Notes in Math., Vol. 1326, Springer, Berlin, 1988, 161-181. 